\title{
Modeling of Turbulent Free Shear Flows
}

Dennis A. Yoder, James R. DeBonis, and Nicholas J. Georgiadis

Glenn Research Center, Cleveland, Ohio 


\section{NASA STI Program . . . in Profile}

Since its founding, NASA has been dedicated to the advancement of aeronautics and space science. The NASA Scientific and Technical Information (STI) program plays a key part in helping NASA maintain this important role.

The NASA STI Program operates under the auspices of the Agency Chief Information Officer. It collects, organizes, provides for archiving, and disseminates NASA's STI. The NASA STI program provides access to the NASA Aeronautics and Space Database and its public interface, the NASA Technical Reports Server, thus providing one of the largest collections of aeronautical and space science STI in the world. Results are published in both non-NASA channels and by NASA in the NASA STI Report Series, which includes the following report types:

- TECHNICAL PUBLICATION. Reports of completed research or a major significant phase of research that present the results of NASA programs and include extensive data or theoretical analysis. Includes compilations of significant scientific and technical data and information deemed to be of continuing reference value. NASA counterpart of peer-reviewed formal professional papers but has less stringent limitations on manuscript length and extent of graphic presentations.

- TECHNICAL MEMORANDUM. Scientific and technical findings that are preliminary or of specialized interest, e.g., quick release reports, working papers, and bibliographies that contain minimal annotation. Does not contain extensive analysis.

- CONTRACTOR REPORT. Scientific and technical findings by NASA-sponsored contractors and grantees.
- CONFERENCE PUBLICATION. Collected papers from scientific and technical conferences, symposia, seminars, or other meetings sponsored or cosponsored by NASA.

- SPECIAL PUBLICATION. Scientific, technical, or historical information from NASA programs, projects, and missions, often concerned with subjects having substantial public interest.

- TECHNICAL TRANSLATION. Englishlanguage translations of foreign scientific and technical material pertinent to NASA's mission.

Specialized services also include creating custom thesauri, building customized databases, organizing and publishing research results.

For more information about the NASA STI program, see the following:

- Access the NASA STI program home page at http://www.sti.nasa.gov

- E-mail your question to help@sti.nasa.gov

- Fax your question to the NASA STI Information Desk at 443-757-5803

- Phone the NASA STI Information Desk at 443-757-5802

- Write to: STI Information Desk NASA Center for AeroSpace Information 7115 Standard Drive Hanover, MD 21076-1320 


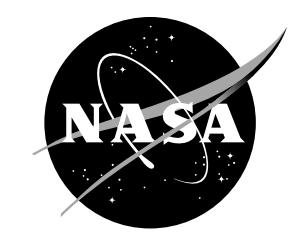

\section{Modeling of Turbulent Free Shear Flows}

Dennis A. Yoder, James R. DeBonis, and Nicholas J. Georgiadis

Glenn Research Center, Cleveland, Ohio

Prepared for the

43rd Fluid Dynamics Conference and Exhibit

sponsored by the American Institute of Aeronautics and Astronautics

San Diego, California, June 24-27, 2013

National Aeronautics and

Space Administration

Glenn Research Center

Cleveland, Ohio 44135 
Level of Review: This material has been technically reviewed by technical management.

Available from

NASA Center for Aerospace Information 7115 Standard Drive

Hanover, MD 21076-1320
National Technical Information Service 5301 Shawnee Road Alexandria, VA 22312

Available electronically at http://www.sti.nasa.gov 


\title{
Modeling of Turbulent Free Shear Flows
}

\author{
Dennis A. Yoder, James R. DeBonis, and Nicholas J. Georgiadis \\ National Aeronautics and Space Administration \\ Glenn Research Center \\ Cleveland, Ohio 44135
}

The modeling of turbulent free shear flows is crucial to the simulation of many aerospace applications, yet often receives less attention than the modeling of wall boundary layers. Thus, while turbulence model development in general has proceeded very slowly in the past twenty years, progress for free shear flows has been even more so. This paper highlights some of the fundamental issues in modeling free shear flows for propulsion applications, presents a review of past modeling efforts, and identifies areas where further research is needed. Among the topics discussed are differences between planar and axisymmetric flows, development versus self-similar regions, the effect of compressibility and the evolution of compressibility corrections, the effect of temperature on jets, and the significance of turbulent Prandtl and Schmidt numbers for reacting shear flows. Large eddy simulation greatly reduces the amount of empiricism in the physical modeling, but is sensitive to a number of numerical issues. This paper includes an overview of the importance of numerical scheme, mesh resolution, boundary treatment, sub-grid modeling, and filtering in conducting a successful simulation.

speed of sound

energy thickness

$10 \% \Delta u$ thickness

turbulence anisotropy tensor, $b_{i j}=\left(-\tau_{i j}^{T}-\frac{2}{3} \bar{\rho} k \delta_{i j}\right) / 2 \bar{\rho} k$

jet nozzle diameter

internal energy

enthalpy

turbulent kinetic energy

acoustic Mach number, $U_{j e t} / a_{\infty}$

convective Mach number, Eq. (9)

gradient Mach number, $|S| l / a$

jet Mach number, $U_{j e t} / a_{j e t}$

turbulent Mach number, $\sqrt{2 k} / a$

pressure

Prandtl number

heat flux

rotation rate tensor

velocity ratio, $u_{2} / u_{1}$

radial location where velocity is half of centerline value

strain rate tensor

density ratio, $\rho_{2} / \rho_{1}$

Schmidt number

temperature

time

axial velocity

convective velocity, Eq. (8)

jet mean velocity

velocity

axial coordinate 


$\begin{array}{ll}x_{c} & \text { potential core length shifted to align peak centerline turbulence values } \\ x_{i} & \text { spatial coordinates } \\ x_{W} & \text { potential core length given by Witze relation } \\ \delta_{i j} & \text { Kronecker delta } \\ \delta_{\text {pit }} & \text { pitot thickness } \\ \delta_{v i s} & \text { visual thickness } \\ \delta_{\omega} & \text { vorticity thickness } \\ \epsilon & \text { turbulent dissipation rate } \\ \epsilon_{d} & \text { dilatation dissipation } \\ \lambda_{s} & \text { velocity-density parameter } \\ \mu & \text { viscosity } \\ \omega & \text { specific turbulent dissipation rate } \\ \Pi^{d i l} & \text { pressure dilatation, } \Pi^{d i l}=\frac{1}{2} \Pi_{i j} \delta_{i j} \\ \Pi_{i j} & \text { pressure-strain correlation tensor, } \Pi_{i j}=\bar{P}^{\prime}\left(u_{i, j}^{\prime \prime}+u_{j, i}^{\prime \prime}\right) \\ \mathcal{P} & \text { production of turbulent kinetic energy, } \mathcal{P}=\tau_{i j}^{T} \widetilde{u}_{i, j} \\ \rho & \text { density } \\ \sigma & \text { spread rate parameter } \\ \tau_{i j} & \text { stress tensor } \\ \tau & \text { turbulent time scale } \\ \theta & \text { momentum thickness } \\ \zeta & \text { turbulent enstrophy } \\ \text { Subscripts } / \text { Superscripts }\end{array}$

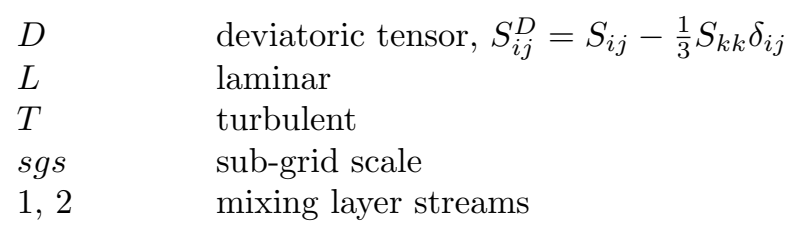

Operators

$\begin{array}{ll}\{\} & \text { trace of the contained tensor expression } \\ \bar{u} & \text { RANS time averaged or LES spatial filtered velocity } \\ \widetilde{u} & \text { RANS density-weighted time average or LES density-weighted spatial filtered velocity } \\ u^{\prime} & \text { RANS fluctuating velcotiy } \\ u^{\prime \prime} & \text { RANS density-weighted Favre-fluctuating velocity }\end{array}$

\section{Introduction}

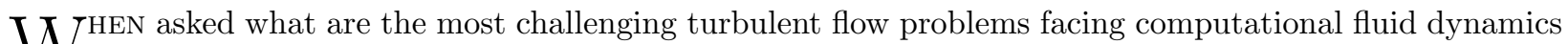
(CFD) for aerospace applications, those involved in model development and application would probably identify one of many wall bounded issues. These include the difficulty in predicting adverse pressure gradient flows, flow separation, and reattachment; streamline curvature; corner flows; shock wave boundary layer interaction; turbulence transition; and heat transfer. Each of these contribute to the ability to predict aerodynamic drag, engine inlet performance metrics such as pressure recovery, and thermal loading. However, turbulent free shear flows such as mixing layers, jets, and wakes also play an important role in aeropropulsion applications. These types of flow involve the motion of fluid that is away from solid surfaces.

Modern subsonic commercial aircraft are typically configured with high-bypass ratio turbofan engines in which the bypass fan stream mixes with the high energy exhaust flow of the core engine. This mixing may occur in the aft portion of the nozzle or in the plume region. The level of noise generated by these exhaust flows increases non-linearly with jet velocity and correlates with the turbulent kinetic energy in the shear layer. Many concepts for reducing jet noise have been investigated over the past several years and resulted 
in significant reductions in jet noise relative to two decades ago. Lobed mixers, chevron nozzles, tabs, fluidic injection, and plasma actuators are some of the concepts that have been explored to increase mixing of the exhaust streams and modify the turbulence characteristics in the jet. Other concepts have been designed to modify the directivity of the noise away from ground observers. This is accomplished by offsetting the core and bypass streams or inserting flow deflection devices into the bypass stream in order to divert more of the lower-energy fan flow below the core stream.

With regulations on aircraft noise becoming ever more stringent, the ability to predict and potentially modify the flowfield to eliminate these sources has been an active research area. Tools based upon an acoustic analogy use the mean flow and turbulence fields from CFD solutions of the Reynolds-averaged Navier-Stokes (RANS) equations to estimate noise levels. Alternatively, large-eddy simulation (LES) can be used to directly compute the unsteady pressure fluctuations in the jet. In these simulations, prediction of both the mean flow and turbulence fields are needed in order to assess the noise of the exhaust nozzle.

Knowledge of the spreading rate of the exhaust flow is also needed in order to address potential plume interaction with the control surfaces of the aircraft and to assess the vulnerability of military aircraft to infrared plume signature detection. For air-breathing hypersonic vehicles, the size and weight of the supersonic combustion ramjet (scramjet) are driven by how rapidly the fuel and oxidizer can be mixed to enable complete combustion.

Wake flows are another important type of free shear flow. As aircraft approach for landing, the unsteady wake generated by the landing gear and control surfaces of the wing generate considerable noise. As with jets, wakes can also have undesirable effects on the downstream control surfaces of the vehicle. In turbomachinery applications, the close proximity of the rotor/stator stages can lead to blade row interaction as the wake from one blade row impacts the next one downstream.

In all of these applications, computational fluid dynamics is a critical tool to be used in evaluating future concepts. In order to predict the viability and efficiency of these systems one must be able to accurately predict turbulent free shear flows. However, simulation of these flows remains a challenge for current CFD methods. RANS-based techniques have been used to reproduce trends that occur due to changing geometry or flow conditions, but their accuracy is still lacking. This is due, in part, to the fact that most RANS models have been developed and calibrated for highly idealized free shear flows and/or the prediction of boundary layers. Modifications intended to correct certain model deficiencies have largely been empirical in nature, not broadly applicable, and in some cases based upon physical arguments that have since been shown to be incorrect. LES and hybrid RANS/LES techniques have demonstrated promise for improving the predictive capability for free shear flows. However, there are still a number of uncertainties in such methods that prevent their use on a regular basis by the aerodynamics community.

This paper reviews the current physical modeling capability for two basic free shear flows, namely mixing layers and jets, which are similar in nature. These "simple" flows involve more than just a mean shear and often display well-organized turbulent structures. The discussion begins with the theory and experimental observations of canonical incompressible mixing layers and jets, followed by the changes that are known to occur in higher-speed compressible flows more applicable to propulsion applications. Specific modeling challenges for both RANS and LES are reviewed, and some suggestions for future research are made.

\section{I.A. Mixing Layers}

Planar mixing layers form when two parallel streams initially separated by a solid surface come into contact. Velocity differences between the two streams create a shear layer, which grows in thickness with downstream distance. Far downstream the flow reaches a state where the profiles of mean velocity and turbulence statistics achieve self-similarity when plotted using appropriately scaled variables. Generally, the mean velocity becomes self-similar before the turbulence quantities, which develop progressively slower in the order of streamwise turbulence intensity, transverse turbulence intensity, then turbulent shear stress. ${ }^{1}$ The shape of the experimentally measured mean velocity profile is often compared with the theoretical error function profile derived by Görtler. ${ }^{2}$

$$
u^{*} \equiv \frac{u-u_{2}}{u_{1}-u_{2}}=\frac{1}{2}\left[1+\operatorname{erf}\left(\eta-\eta_{0}\right)\right]
$$

where the similarity variable $\eta$ is defined as

$$
\eta=\sigma \frac{y}{x-x_{0}}
$$


To use this expression, three parameters must be known or determined experimentally: $\sigma$, the spread rate parameter; $x_{0}$, the location of the virtual origin of the mixing layer, which is often upstream of the physical location where the mixing layer begins; and $y_{0}$ (or $\eta_{0}$ ), the transverse location of the center of the shear layer. The spread rate parameter can be related to the velocity ratio $(r)$ and density ratio $(s)$ of the two streams, ${ }^{3}$

$$
\frac{\sigma_{0}}{\sigma}=\lambda_{s} \equiv \frac{(1-r)(1+\sqrt{s})}{2(1+r \sqrt{s})}
$$

and a value of $\sigma_{0}=11$ is often used.

From a practical perspective, there is no way to measure the exact width of the unsteady shear layer. Therefore, researchers have devised various measures of the shear layer width which are readily quantifiable and can be related to the the spread parameter. One measure of the shear layer width is the visual thickness $\delta_{v i s}$ obtained from images of the flow. The spreading angles of both sides of the mixing layer are estimated by drawing straight-line mean tangents to the "edges" of the mixing layer. The visual thickness at any given axial location is then defined as the distance between the edge lines. As a more objective measure, researchers have used thickness definitions based upon profiles of pitot pressure or mean velocity. The various velocitybased thickness definitions include: the $10 \% \Delta u$ thickness, $b$; the vorticity thickness, $\delta_{\omega}$; the momentum thickness, $\theta$; and the energy thickness, $B$. Samimy and Elliot ${ }^{4}$ discuss some of the benefits and shortcomings of these various measures of the mixing layer thickness, particularly with regards to: (1) the number and reliability of data points used in describing the thickness, and (2) the ability of that parameter to describe variations in the mixing layer thickness due to changes in flow characteristics.

In the fully-developed region, the mixing layer thickness is found to grow linearly with downstream distance. Using equations (1-3), the growth rates can be related to the velocity-density parameter, $\lambda_{s}$.

$$
\frac{d b / d x}{0.165}=\frac{d \delta_{\omega} / d x}{0.161}=\frac{d \theta / d x}{0.036}=\frac{d B / d x}{0.135}=\lambda_{s}
$$

Figure 1 shows that Eq. (4) predicts the growth rate fairly well, except for the last few data sets that involve streams with differing densities. Empirical relations for the mixing layer growth rate have also been put forth. ${ }^{3,5}$

$$
\frac{d \delta_{p i t} / d x}{0.28}=\frac{d \delta_{v i s} / d x}{0.34}=\frac{d \delta_{\omega} / d x}{0.17}=\lambda_{s}
$$

These relations are based upon experimental data rather than Görtler's velocity profile.

\section{I.A.1. Experimental Data}

Similarity profiles of mean velocity and turbulence quantities from a number of incompressible mixing layer experiments are presented in figure 2. The shape of the experimentally measured mean velocity profiles compares very well with Görtler's error function profile. The turbulence profiles all exhibit very similar Gaussian-like curves, which are in line with that obtained analytically for the shear stress when a simple eddy viscosity model is used. ${ }^{6,32}$

$$
\frac{-\overline{u^{\prime} v^{\prime}}}{(\Delta U)^{2}}=\frac{1}{4 \sqrt{\pi} \sigma_{0}} \exp \left[-\left(\eta-\eta_{0}\right)^{2}\right]
$$

Peak values of the normalized turbulence quantities are found to lie within the approximate ranges:

$$
\begin{aligned}
0.010 \leq-\overline{u^{\prime} v^{\prime}} /(\Delta U)^{2} & \leq 0.013 \\
0.025 \leq \overline{u^{\prime} u^{\prime}} /(\Delta U)^{2} & \leq 0.034 \\
0.016 \leq \overline{v^{\prime} v^{\prime}} /(\Delta U)^{2} & \leq 0.020 \\
0.020 \leq \overline{w^{\prime} w^{\prime}} /(\Delta U)^{2} & \leq 0.022 \\
0.032 \leq \quad k /(\Delta U)^{2} & \leq 0.035
\end{aligned}
$$

According to the data of $\mathrm{Mehta}^{25}$ the normalized turbulence quantities exhibit no discernible trends with increasing velocity ratio. However, elevated freestream turbulence levels, such as in the case of Pui and 


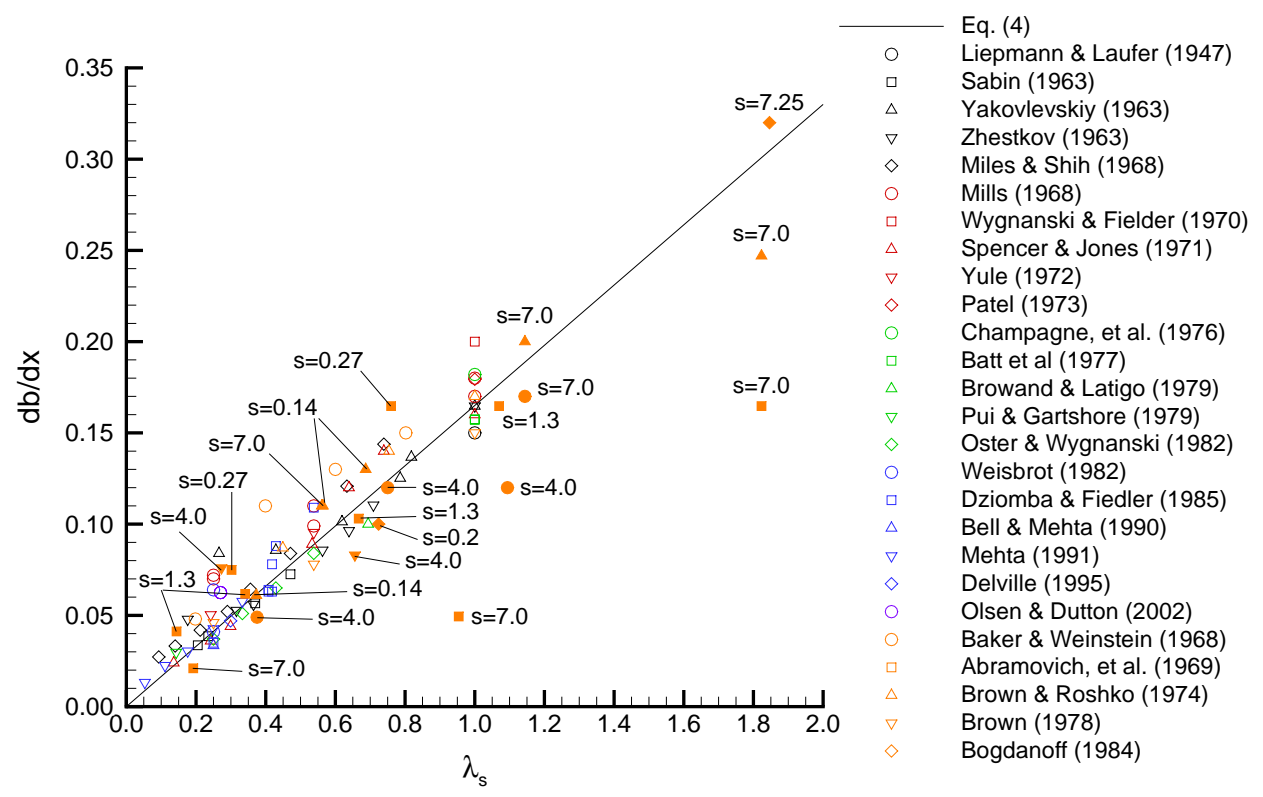

Figure 1: Incompressible mixing layer growth rates from reference 6 . Solid symbols and labels indicate density ratios other than 1.0.

Gartshore ${ }^{20}$ can lead to higher peak turbulence values within the mixing layer and an increase in the mixing layer growth rate. ${ }^{23,32}$ References 35 and 24 investigated the effect of the boundary layer state upstream of the mixing layer and found that when laminar boundary layers are present the growth rate and Reynolds stresses are significantly higher in the development region than when the boundary layers are turbulent. Far downstream the turbulence values for both cases asymptote to approximately the same constant values. Champagne ${ }^{17}$ conjectures that if the initial boundary layer is turbulent, then the orderly vortex-pairing process (described in the next section) might occur randomly, in some intermittent fashion, or not at all, and therefore lead to subsequent differences within the development region.

\section{I.A.2. Structure}

In incompressible turbulent mixing layers, large-scale spanwise-oriented vortical structures have been observed. $^{5}$ The formation of these large-scale structures is triggered by the fundamental Kelvin-Helmholtz instability of the flow, and it is the exponential growth of these instabilities which causes the shear layer to roll-up into large spanwise vortices. The initial spacing between vortices is tied to the frequency of the most dominant instabilities, as evidenced when forcing is applied to the mixing layer.

These spanwise rollers convect downstream at roughly the average speed of the two streams. As they do, they grow by entraining fluid from outside the shear layer and they combine through a series of pairing and tearing processes. ${ }^{36}$ In the pairing process, two adjacent eddies draw together and begin rotating about each other, redistributing their vorticity until they eventually merge into a single larger eddy. This pairing process may be triggered by local instabilities which push one of the eddies away from the centerline, thereby giving it a slightly faster or slower convective velocity depending on which stream it is pushed towards. In the tearing process, a portion of an eddy splits apart from the rest and is consumed by another neighboring eddy. This amalgamation of eddies through the pairing and tearing processes results in fewer, larger structures. Therefore, the spacing between eddies increases with distance downstream.

As the flow progresses downstream, secondary spanwise instabilities are also triggered which lead to the development of additional streamwise vortices. ${ }^{37}$ These counter-rotating streamwise vortices or "ribs" form in the braid region between rollers and wrap around successive spanwise vortices. ${ }^{38,39}$ The spanwise spacing between these streamwise vortices is observed to increase further downstream and may be related to the increasing streamwise spacing of the larger rolling structures. ${ }^{40}$ Jimenez, Cogollos, and Bernal ${ }^{39}$ estimate that the strength of the streamwise ribs can be a significant fraction of the local circulation in the 


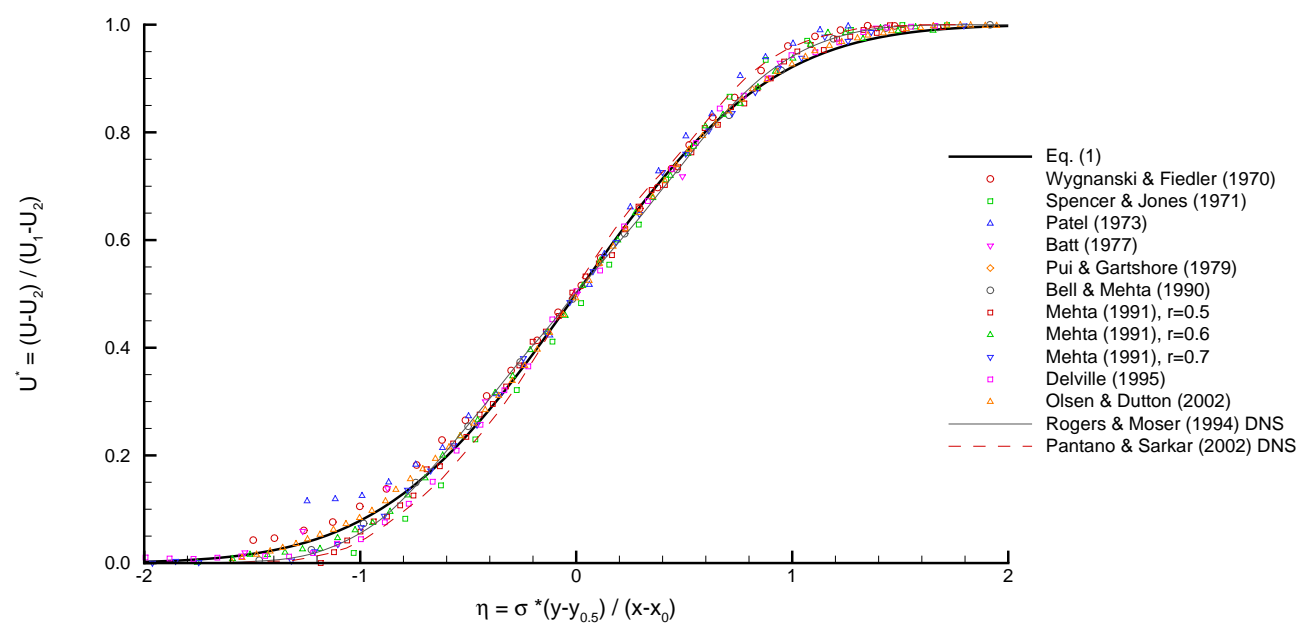

(a) Mean Velocity

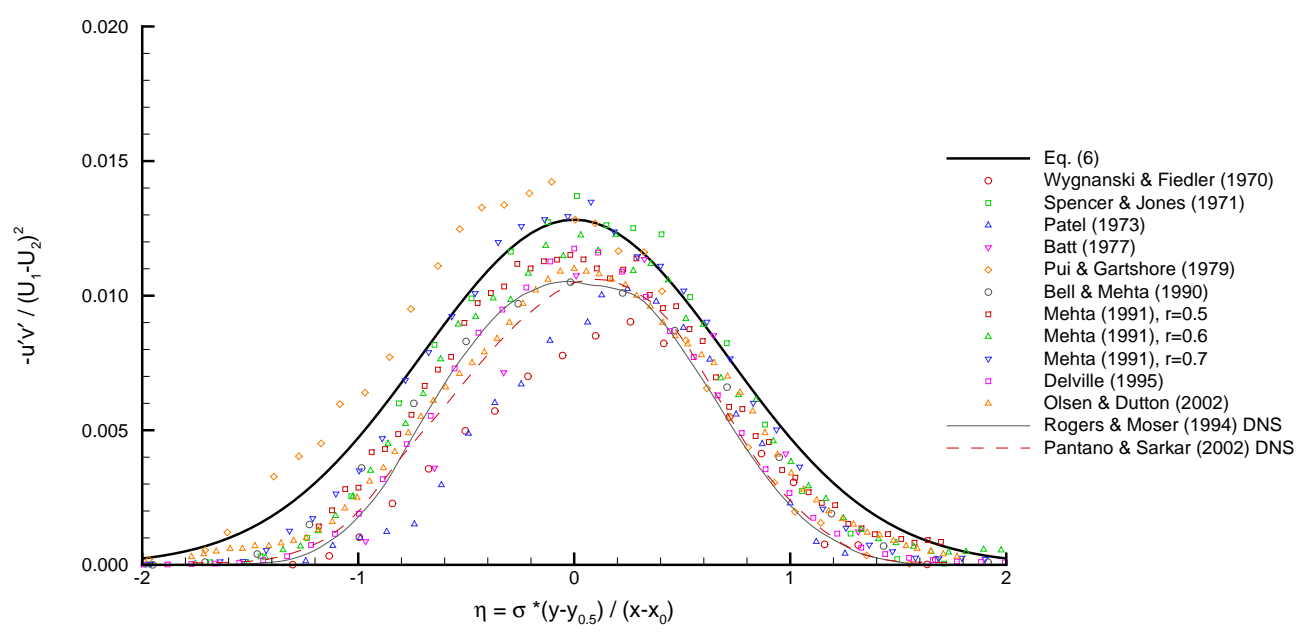

(b) Shear Stress

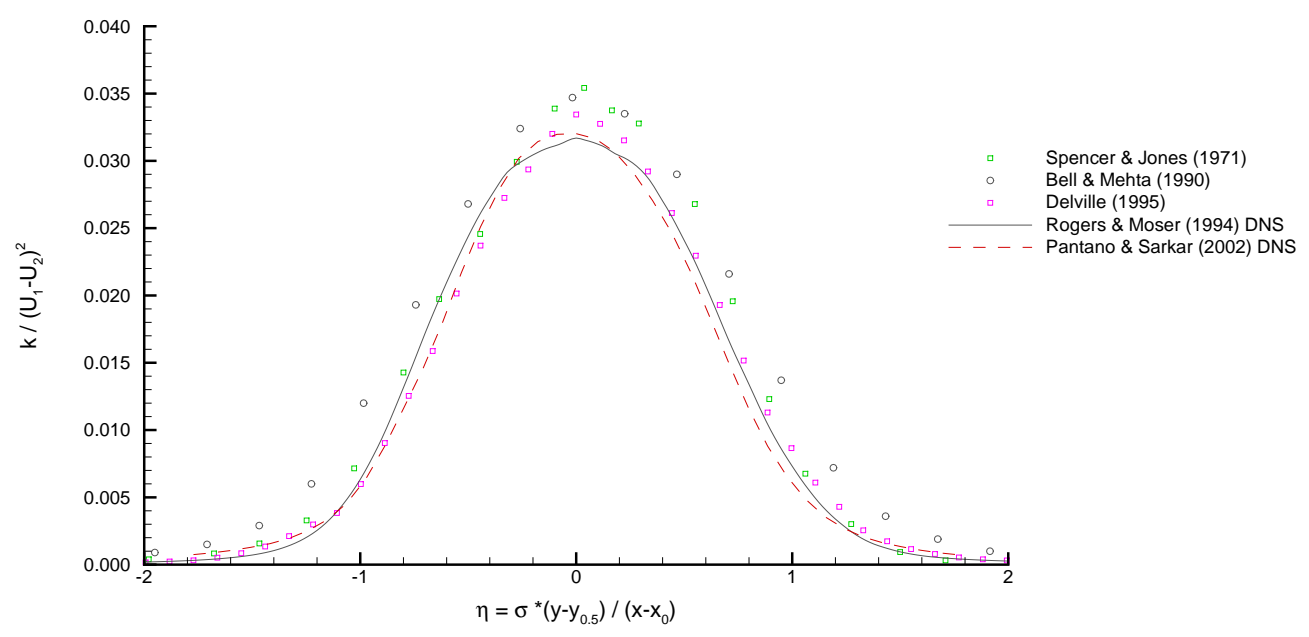

(c) Turbulent Kinetic Energy

Figure 2: Similarity profiles of incompressible mixing layers from reference 6 . 


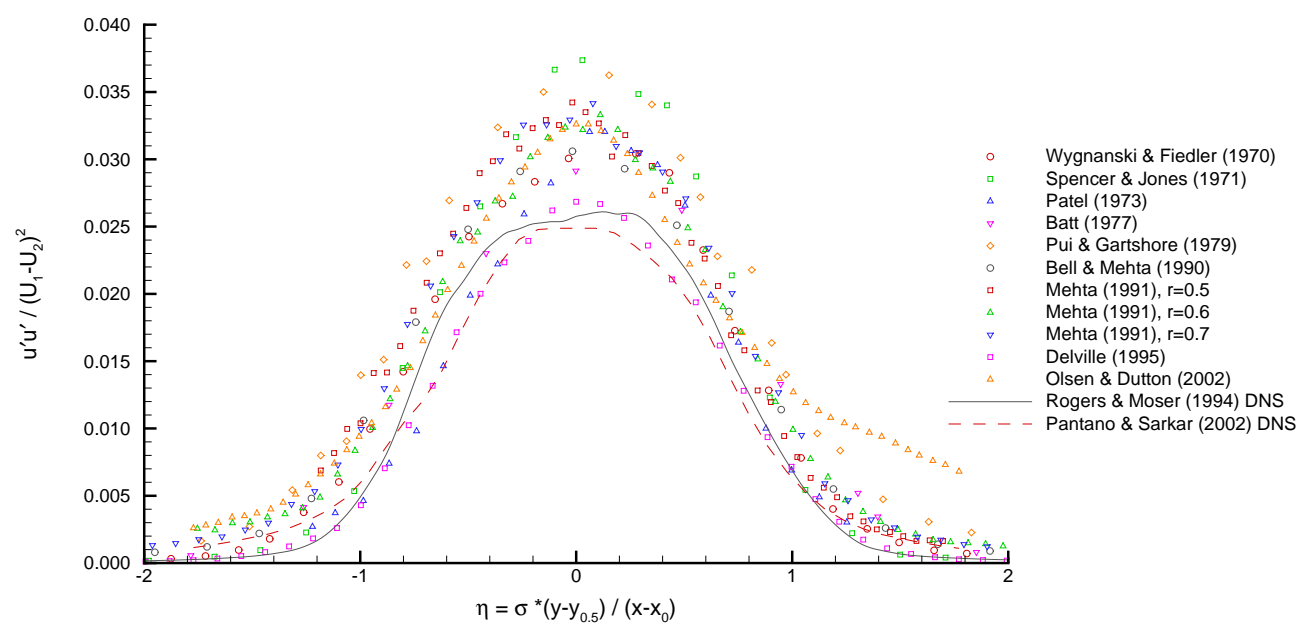

(d) Streamwise Normal Stress

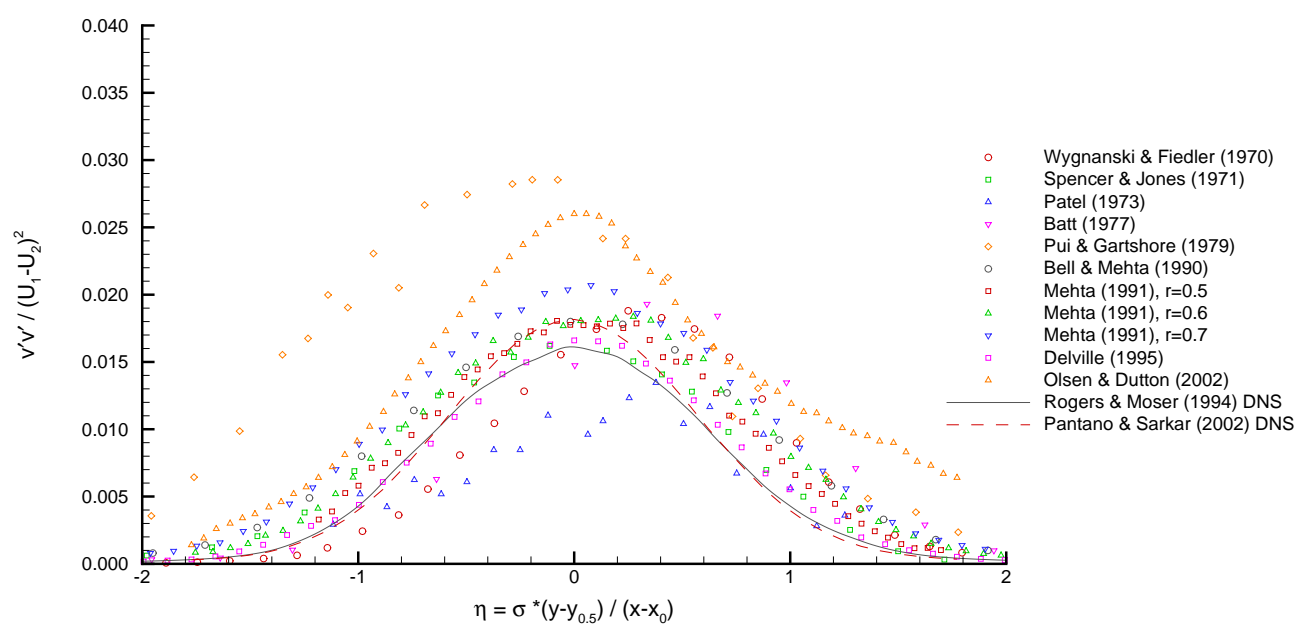

(e) Transverse Normal Stress

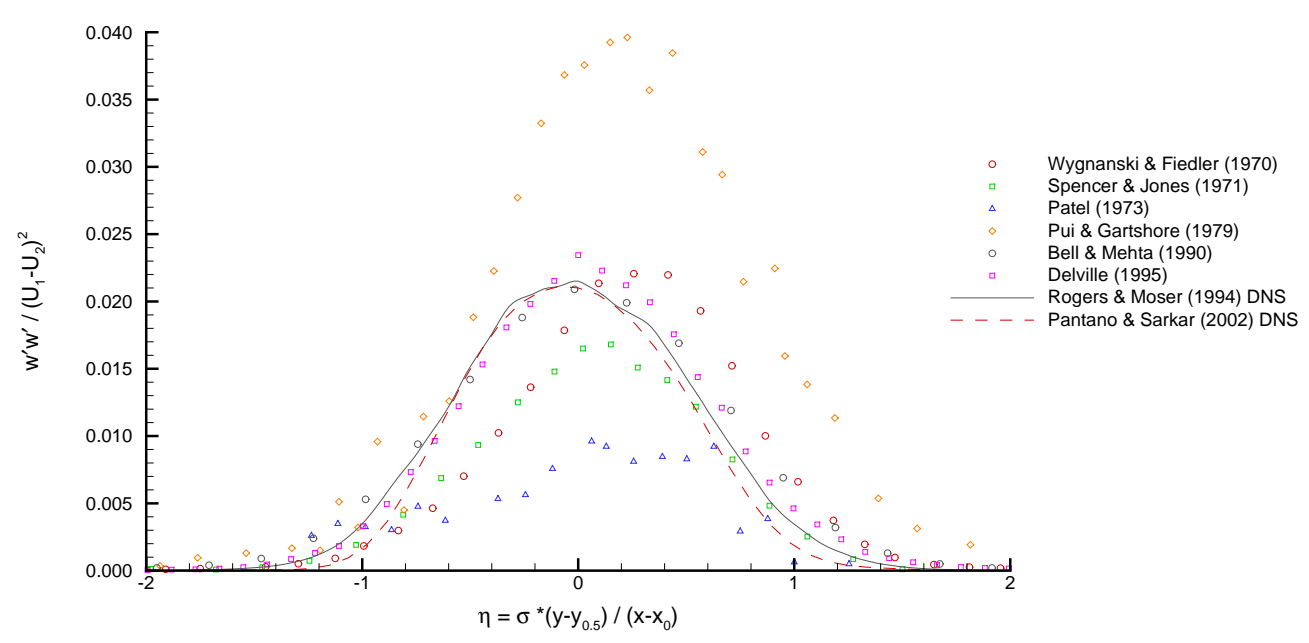

(f) Spanwise Normal Stress

Figure 2: Similarity profiles of incompressible mixing layers from reference 6 (continued). 
spanwise eddies. The interaction of these structures causes a distortion of the large-scale spanwise rollers, three-dimensionality, and vortex stretching. These factors work to feed higher-order instabilities which lead to the development of smaller scale turbulence. The energy of the large turbulent eddies cascades to progressively smaller eddies until it reaches the smallest Kolmogorov scales where it is then dissipated into heat by molecular viscosity. This action leads to the eventual decay of all of the turbulent structures.

\section{I.A.3. Compressibility}

One of the key observations of compressible planar mixing layers is that they tend to spread more slowly than incompressible mixing layers at equivalent velocity and density ratios. Since many of the early experiments investigated single-stream mixing layers, where one stream was at rest and increasing the Mach number of the other was accompanied by decreasing temperature and therefore increasing density, this reduction in growth rate was generally thought to be caused by density differences between the two streams. However, subsequent dual-stream experimental results ${ }^{5}$ revealed that the effect of extreme density differences alone could not explain this phenomenon and that the reduction in growth rate of compressible mixing layers is a true compressibility effect.

The convective Mach number is one of the most commonly used measures for quantifying the level of compressibility of mixing layer flows. For two pressure-matched streams with the same ratio of specific heats, the convective velocity and Mach number are computed as

$$
\begin{gathered}
U_{c}=\frac{a_{2} u_{1}+a_{1} u_{2}}{a_{1}+a_{2}} \\
M_{c}=\frac{u_{1}-u_{2}}{a_{1}+a_{2}}
\end{gathered}
$$

and represent the rate at which the large-scale structures propagate downstream.

Using the convective Mach number as a measure of compressibility, the mixing layer growth rates from a variety of experimental data sets are summarized as shown in figure 3 . These growth rates have been normalized by the correlation for incompressible mixing layer growth rate at the same velocity and density ratios. The figure indicates a clear pattern of reduced growth rate with increasing compressibility, at least until $M_{c}>1.0$ where additional compressibility seems to have only a minimal effect. The scatter in the data has been attributed to a number of factors, including: the use of different experimental facilities and measurement techniques, the use of data which has not fully achieved self-similarity, differences in the mixing layer thickness definition used, uncertainty in the estimate of the incompressible mixing layer thickness, and the possibility that the convective Mach number may not fully capture the effects of compressibility or may only capture the first-order effects. ${ }^{38}$ In particular, the data of figure 3 indicate a disparity between mixing layer growth rate measurements based on pitot thickness or visual thickness (shown as solid symbols) and the various other growth rate estimates which are based on velocity measurements.
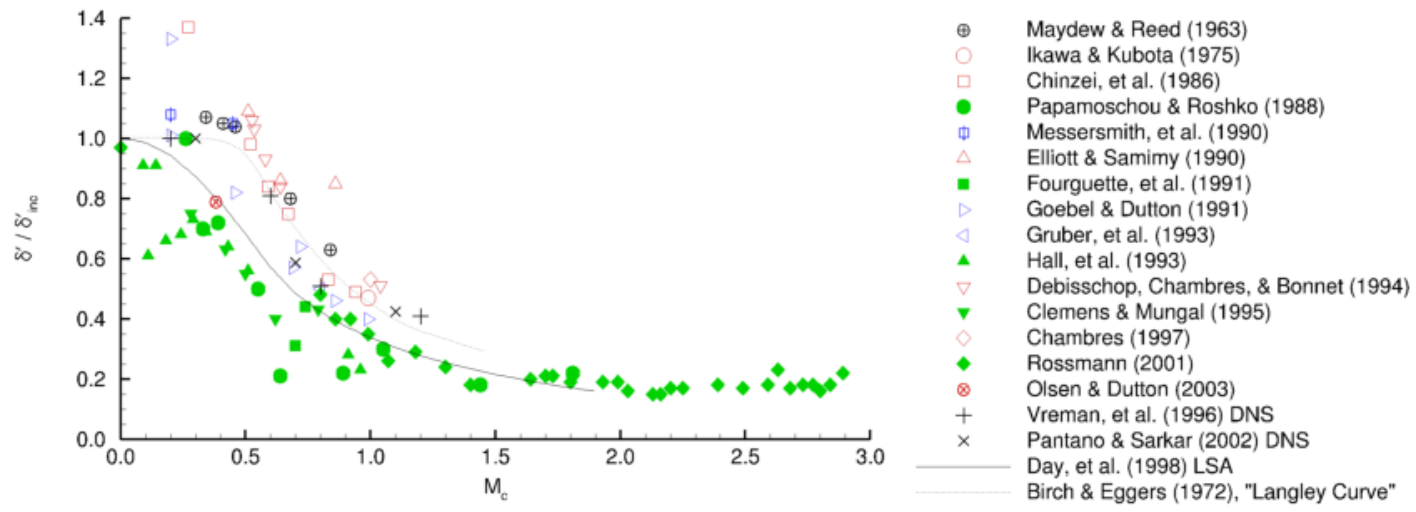

Figure 3: Normalized compressible mixing layer growth rate data from reference 6.

Figure 4 compares available Reynolds stress data for compressible mixing layers. There is a significant reduction in the shear stress and transverse normal stress with increasing convective Mach number. These changes in turbulence statistics occur at convective Mach numbers as low as 0.2 , well before any reduction in the corresponding mixing layer growth rate data is observed $\left(M_{c} \approx 0.5\right)$. Trends in the data for the 
streamwise turbulence intensity are not as straightforward. The measurements of Goebel and Dutton ${ }^{1}$ and Gruber, et al. ${ }^{48}$ indicate that the streamwise turbulence remains relatively unaffected by compressibility. Others have noted a slight reduction, though not as severe as with the other turbulence quantities. This disparity in the measured change in turbulence anisotropy is quite evident by plotting the ratio $\overline{u^{\prime} u^{\prime}} / \overline{v^{\prime} v^{\prime}}$ versus convective Mach number, as done in figure $4 \mathrm{~d}$.

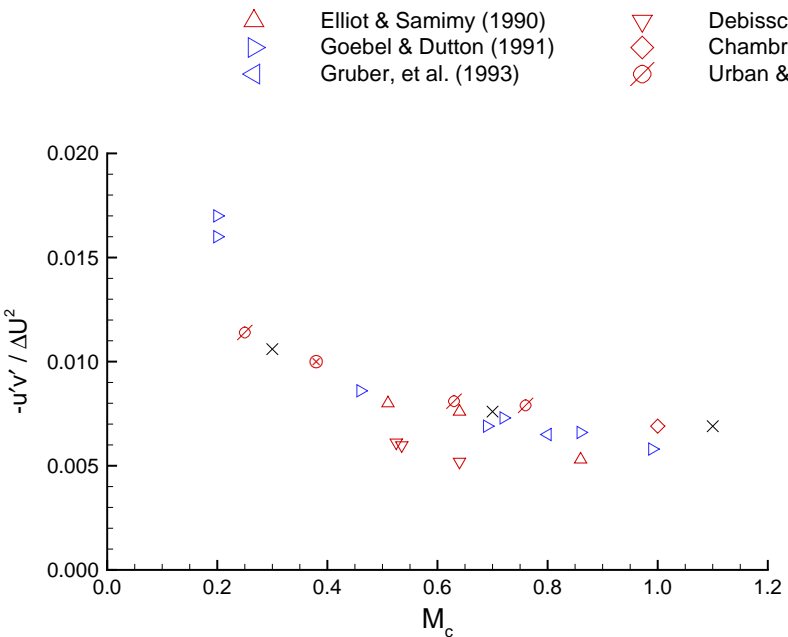

(a) Shear Stress

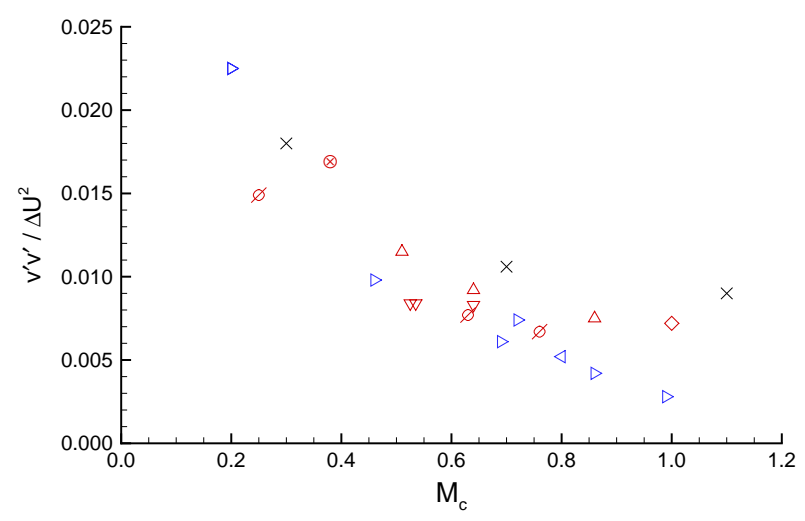

(c) Transverse Normal Stress
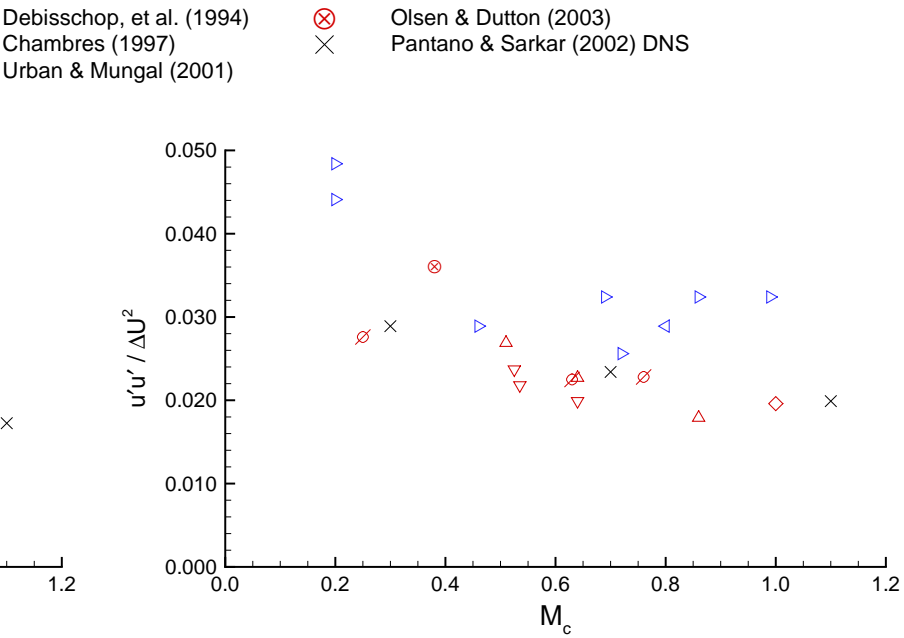

(b) Streamwise Normal Stress

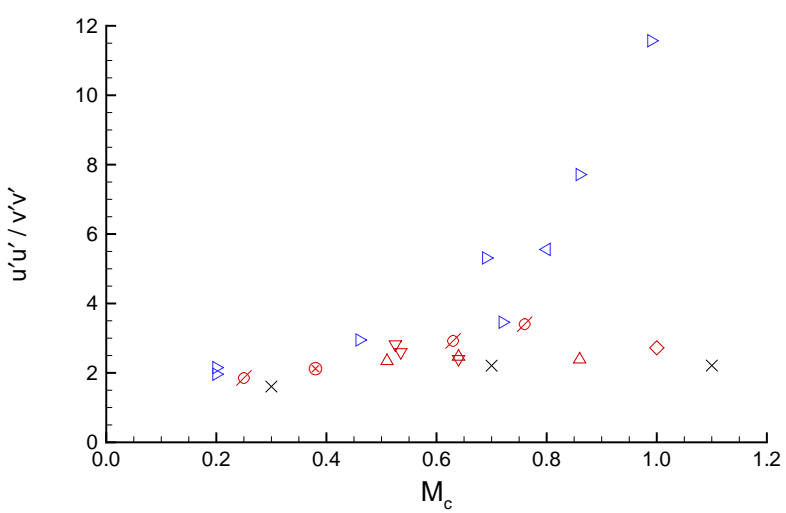

(d) Turbulence Anisotropy

Figure 4: Turbulent stress values for compressible mixing layers from reference 6.

Along with a reduction in mixing layer growth rate and turbulence statistics, structural changes have also been observed in compressible mixing layers. ${ }^{58,59}$ At low convective Mach numbers, large-scale vortical structures are sometimes observed to extend spanwise across the width of the test section much like they do in incompressible flows. At moderate convective Mach numbers $\left(M_{c} \approx 0.5\right)$ the Brown-Roshko style large-scale organized structures are less frequently encountered and often appear smaller in size. Instead the high- and low-speed interfaces of the shear layer appear less smooth with the freestream fluid sharply intruding into the middle of the shear layer. Plan views indicate that the mixing layer becomes more threedimensional with less observed spatial regularity. At higher convective Mach numbers, the structures are highly three-dimensional and the flows tend to exhibit thin streamwise sheets of vorticity which appear at various transverse locations. These sheets do not extend as far across the mixing layer as the incompressible rolling structures and exhibit little transverse communication among themselves. It has been suggested that this reduced communication ${ }^{60,61}$ across the shear layer results in the reduction of the transverse turbulence intensity and turbulent shear stress, which affects the mixing layer growth rate. 


\section{I.B. Jets}

In jet flows, one fluid stream mixes with a surrounding medium that is either at rest or in motion. Viscous forces along the shared interface between the inner and outer streams result in the formation of a shear layer that transfers momentum between streams. The largely inviscid flow within the inner stream is referred to as the potential core. The shear layer grows with distance downstream until it completely consumes the potential core. For jets issuing from the round nozzles found on many aircraft engines or rocket exhaust systems, the shear layer is circular in shape. Flow in the initial part of the jet may be loosely characterized as a planar-like shear layer, because the width of the shear layer is small compared to the diameter of the jet. Gutmark, Schadow, and $\mathrm{Yu}^{62}$ discuss some of the differences between planar mixing layers and jet flows. In particular they note that round jets support additional axisymmetric (ring-like) and helical modes and that the number of vortex interactions in jets is limited by the introduction of additional length scales, such as the length of the potential core. Linearized stability analysis has demonstrated that both the axisymmetric and helical modes of jets become more stable as the Mach number increases. ${ }^{63}$

Jets from non-circular nozzles support a different set of instability modes which are parametrized by factors such as eccentricity, aspect ratio, circumferential distribution of momentum thickness, and local radius of curvature. ${ }^{64}$ In these flows, shear layer growth and the formation of small-scale turbulence is driven more by the dynamics of vortex self-induction, also known as $\omega_{\theta}$-dynamics, than the vortex pairing process. ${ }^{65}$ Self-induction ${ }^{66}$ refers to the phenomenon whereby the advection velocity of a curved vortex filament is moves faster in regions of greater curvature. For elliptical or rectangular jets, that portion of the azimuthal vortex ring located near either end of the major axis will accelerate out-of-plane. The distortion of the vortex ring causes an induced velocity on the minor-axis sides which pushes the flow outward. After several contortions, the vortex ring becomes planar once again but appears to be rotated by 90 degrees relative to the initial orientation. This axis switching can repeat several times before the vortex breaks downs and decays.

For nozzles that have corners or flow control devices such as tabs, the induced motion of streamwise vortex pairs in the flow, known as $\omega_{x}$-dynamics, is important. ${ }^{67}$ In rectangular nozzles, differences in the cross-stream pressure gradients inside the nozzle result in the formation of secondary flows consisting of streamwise-oriented vortex pairs. The induced motion of these vortex pairs will cause them to move from the corners toward the center of the jet and displace flow towards the sides. This dynamic can likewise lead to an axis switchover, but will not support further switchings beyond the first. Nozzles that have both flat sides and corners benefit from the large-scale mixing that occurs at the flat sides and the small-scale mixing due to the corner flow.

The reduced shear layer growth rate in compressible turbulent jets results in longer potential core lengths. In practice, jets are found to exhibit compressibility effects at jet Mach numbers higher than about 0.5. For unheated incompressible jets the potential core length has been found to be approximately 5 nozzle exhaust diameters. For an unheated Mach 2 jet, the potential core length is approximately 10 nozzle diameters long, twice the value for an incompressible jet.

It has been experimentally observed that for otherwise similar jets, e.g. from the same nozzle at same jet exit Mach number, a heated jet will exhibit faster mixing than the corresponding unheated jet. Lau ${ }^{68}$ and Lepicovsky ${ }^{69}$ concluded that the boundary layer state at the end of nozzle my be largely responsible for the differences in heated jet development. However, other studies such as that of Monkewitz and Sohn ${ }^{70}$ and Monkewitz, et al. ${ }^{71}$ have shown that the instabilities in the initial part of a heated jet give rise to a faster rate of turbulent structure growth and resultant faster jet mixing rate in comparison to unheated jets. They indicate that for jets with temperature ratios greater than 1.4 (or corresponding density ratios less than approximately 0.7 ), a local absolute instability region may develop in the jet potential core which is primarily responsible for this enhanced mixing behavior.

Witze $^{72}$ examined a number of jet experiments to develop expressions for the centerline velocity decay and jet potential core length as a function of jet Mach number and ratio of jet to freestream densities.

$$
\begin{gathered}
\frac{U}{U_{\text {jet }}}=1-\exp \left[\frac{1}{0.70\left(1-x / x_{W}\right)}\right] \\
\frac{x_{W}}{D_{\text {jet }}}=\frac{0.70}{2 \kappa \sqrt{\rho_{\infty} / \rho_{\text {jet }}}}
\end{gathered}
$$


Table 1: Test conditions used in reference 73.

\begin{tabular}{|c|c|c|c|c|}
\hline Set Point & $M_{a}$ & $T_{\text {jet }} / T_{\infty}$ & $\mathrm{NPR}$ & $M_{\text {jet }}$ \\
\hline 3 & 0.500 & 0.950 & 1.197 & 0.513 \\
7 & 0.900 & 0.835 & 1.861 & 0.985 \\
23 & 0.500 & 1.764 & 1.102 & 0.376 \\
27 & 0.900 & 1.764 & 1.357 & 0.678 \\
29 & 1.330 & 1.764 & 1.888 & 1.001 \\
46 & 0.900 & 2.700 & 1.219 & 0.548 \\
49 & 1.485 & 2.700 & 1.678 & 0.904 \\
\hline
\end{tabular}

For subsonic jets,

$$
\kappa=0.08\left(1-0.16 M_{j e t}\right)\left(\rho_{\infty} / \rho_{j e t}\right)^{-0.22}
$$

For properly-expanded supersonic jets,

$$
\kappa=0.063\left(M_{j e t}^{2}-1\right)^{-0.15}
$$

This expression is valid from the nozzle exit to the sonic point. Downstream of that, the subsonic expression can be used provided an appropriate shift in $x$ is made.

Bridges and Wernet ${ }^{73}$ provide a concise yet thorough review of available data for subsonic round jets. They show that normalizing the axial coordinate by Witze's potential core length leads to a satisfactory collapse of low speed axial turbulence data along the jet centerline. They also present their own particle image velocimetry (PIV) data for various nozzles operated over the range of conditions listed in table 1. After careful consideration of the measurement error, they arrive at a consensus data set that represents the best estimate of the flow statistics and uncertainty.

Figure 5 compares the centerline profiles of mean and turbulence axial velocity for all of the consensus data sets. While normalizing by Witze's correlation for potential core length provides good collapse of most of the data, Bridges and Wernet prefer to use a potential core length $x_{c}$ that is deliberately shifted to align the peak turbulence values along the centerline. Doing so results in centerline profiles for the streamwise turbulence that are similar in shape, but increase in peak amplitude with increasing jet temperature.
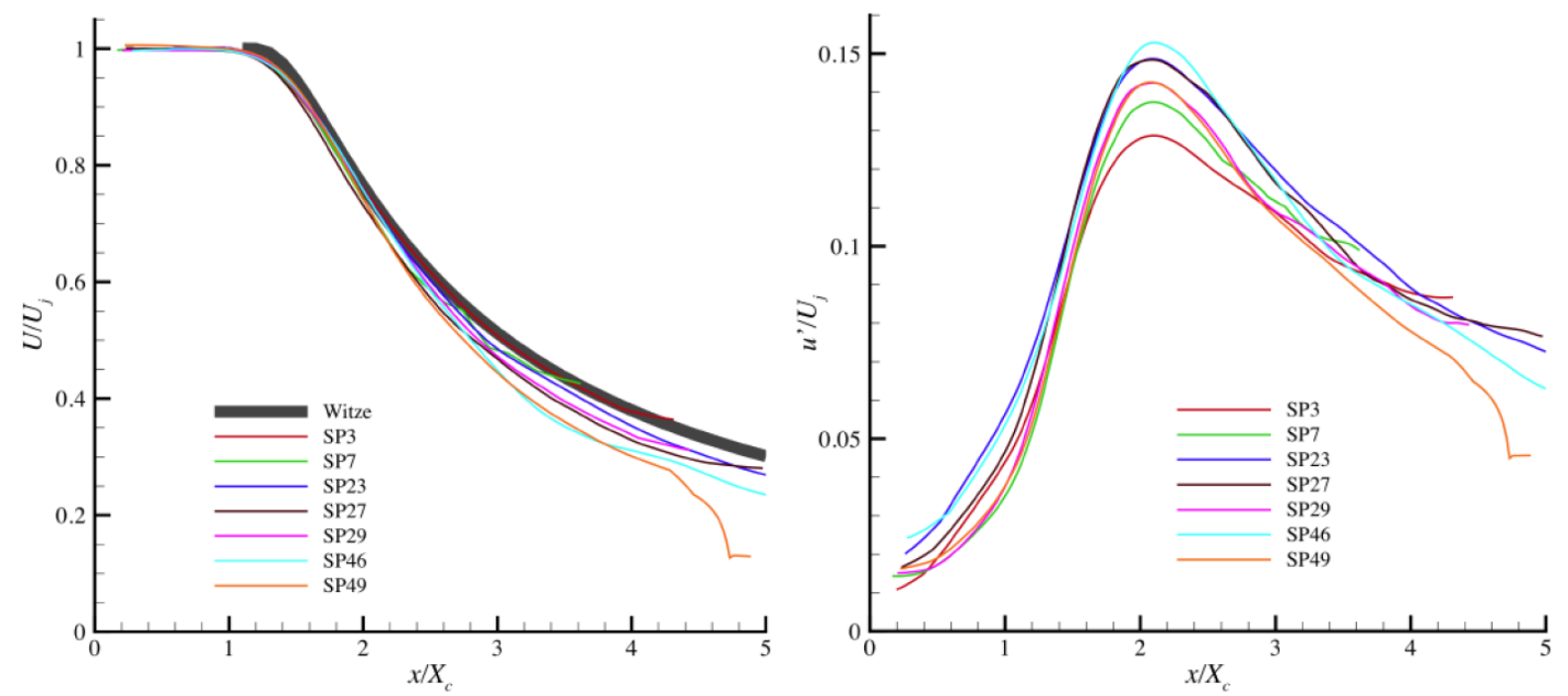

Figure 5: Axisymmetric jet centerline profiles of mean (left) and turbulent (right) axial velocity from reference 73. 
Figure 6 shows the sensitivity of both potential core metrics to temperature and Mach number. The elevated turbulence levels in heated jets results in shorter potential core lengths. As the jet Mach number increases, so do compressibility effects which reduce the jet spread rate and increase the length of the potential core. It is also evident that as the jet Mach number increases, so does the discrepancy between the two potential core metrics. The core length based on peak centerline turbulence value tends to shift farther downstream.

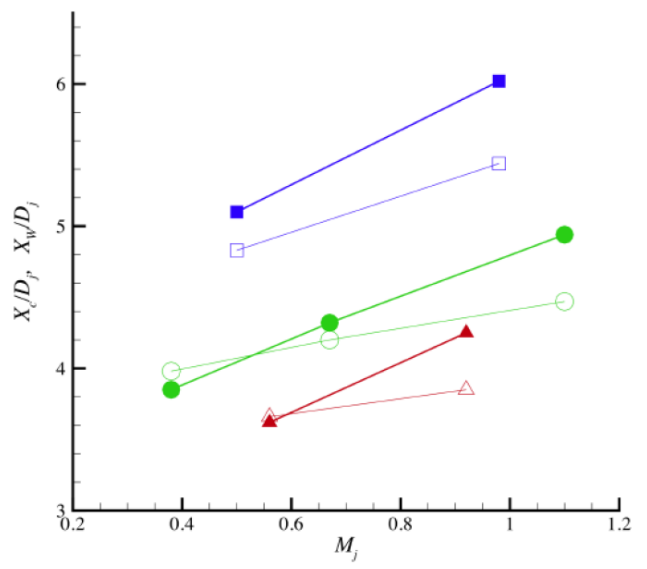

Figure 6: Axisymmetric jet potential core lengths as a function of Mach number for jets with different temperature ratios from reference 73. Squares, unheated; circles, $T_{j e t} / T_{\infty}=1.7$; triangles, $T_{j e t} / T_{\infty}=2.7$. Open symbols are $x_{W} / D_{j e t}$, closed symbols are $x_{c} / D_{j e t}$.

The peak axial turbulent velocity on the centerline and in the entire flowfield is plotted against potential core length in figure 7 . The peak turbulence values do not occur along the centerline, but rather in the shear layer just downstream of the nozzle lip. However the peak centerline value appears to be proportional to the overall peak value. Higher turbulence values result in a more rapid growth of the jet shear layer and shorter potential core length.

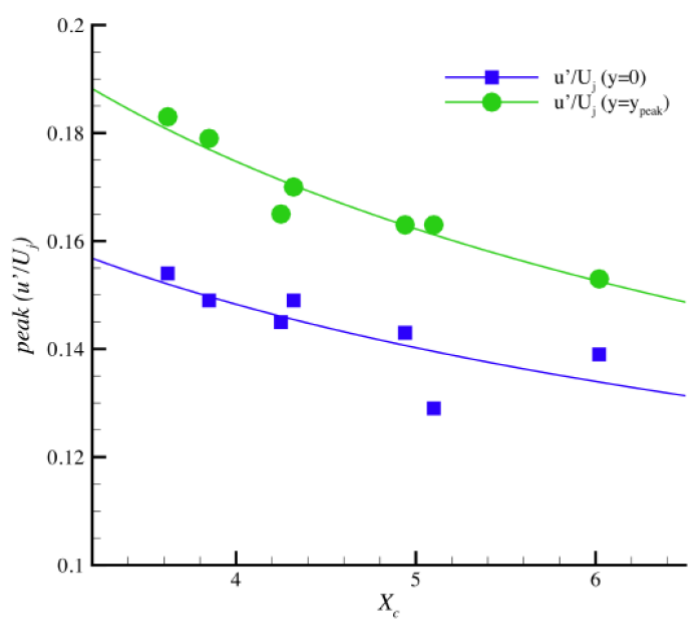

Figure 7: Axisymmetric jet peak axial turbulent velocity on the centerline and throughout the flowfield as a function of potential core length from reference 73.

Radial spreading of the jet is another important parameter. Mean and turbulent axial velocity contours are shown in figure 8. Even though the axial coordinate is scaled by the potential core length, the contours show some variation with static temperature (or density). The heated jets do not appear to extend as far radially and the centerline velocity decays more quickly. Higher turbulence levels are also observed farther downstream and closer to the centerline. Bridges and Wernet show that better collapse of the contours can be made if the radial coordinate is scaled by the jet half-width $\left(r_{0.5}\right)$ rather than nozzle diameter. The half-width, which is a measure of the center of the jet shear layer, is shown to be insensitive to Mach number. 
However, the center of the shear layer for hot jets is shifted closer to the axis. The shear layer growth rate, on the other hand, is sensitive to Mach number but not temperature. For hot jets then, the shear layer is shifted closer to the axis but grows toward the centerline at the same rate as cold jets. The result is a shorter potential core.
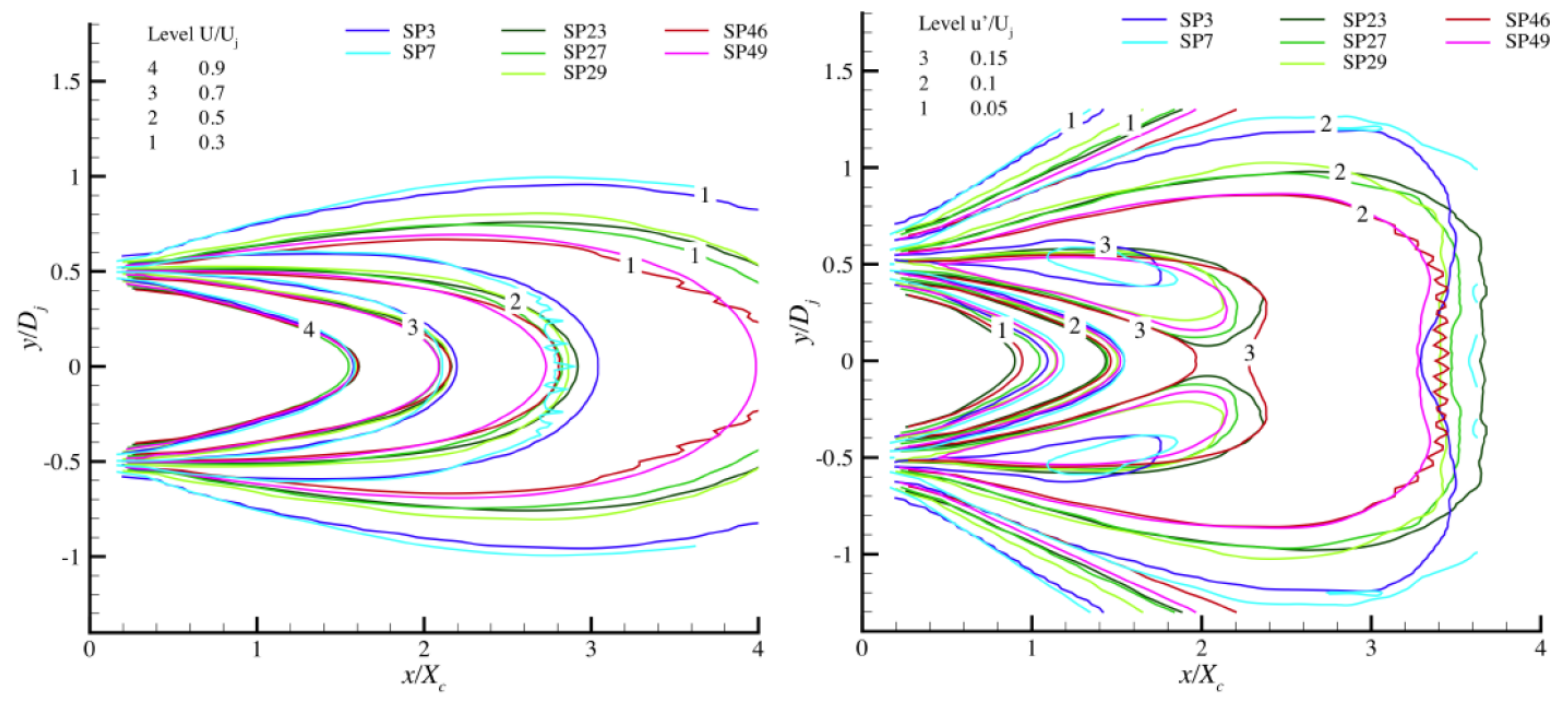

Figure 8: Axisymmetric jet contours for mean (left) and turbulent (right) axial velocity from reference 73.

\section{RANS Modeling Issues}

The Reynolds-averaged Navier-Stokes (RANS) equations are routinely used to simulate aeropropulsion flows. With this method, a time averaging procedure is used to represent the net effect of the turbulent fluctuations without having to simulate the entire unsteady motion. In this respect the method is very efficient. However, the averaging process introduces unknown correlation terms that must be modeled.

The Reynolds averaging procedure decomposes each instantaneous flow variable into a mean and a fluctuation about that mean. For example,

$$
u=\bar{u}+u^{\prime}
$$

where the Reynolds-averaged mean value is defined as

$$
\bar{u} \equiv \lim _{\Delta t \rightarrow \infty} \frac{1}{\Delta t} \int_{t_{o}}^{t_{o}+\Delta t} u d t
$$

and $\Delta t$ represents a long period of time relative to the fluctuations. For compressible flows, a density-weighted procedure leads to a more compact form of the flow equations. Here,

$$
u=\widetilde{u}+u^{\prime \prime}
$$

where the Favre-averaged mean value is defined as $\widetilde{u} \equiv \overline{\rho u} / \bar{\rho}$. The resultant averaged form of the continuity, momentum, and energy equations can be written as:

$$
\begin{gathered}
\frac{\partial \bar{\rho}}{\partial t}+\frac{\partial \bar{\rho} \widetilde{u}_{j}}{\partial x_{j}}=0 \\
\frac{\partial \bar{\rho} \widetilde{u}_{i}}{\partial t}+\frac{\partial}{\partial x_{j}}\left[\widetilde{\rho}_{i} \widetilde{u}_{j}+\bar{P} \delta_{i j}-\left(\bar{\tau}_{i j}+\tau_{i j}^{T}\right)\right]=0 \\
\frac{\partial}{\partial t}\left[\bar{\rho}\left(\widetilde{e}+\frac{1}{2} \widetilde{u}_{i} \widetilde{u}_{i}+k\right)\right]+\frac{\partial}{\partial x_{j}}\left[\bar{\rho} \widetilde{u}_{j}\left(\widetilde{h}+\frac{1}{2} \widetilde{u}_{i} \widetilde{u}_{i}+k\right)-\widetilde{u}_{i}\left(\bar{\tau}_{i j}+\tau_{i j}^{T}\right)+\left(\bar{q}_{j}+q_{j}^{T}\right)\right]=0
\end{gathered}
$$


where the important correlation terms to be modeled are the turbulent heat flux, the turbulent stress tensor, and the turbulent kinetic energy defined as:

$$
\begin{aligned}
q_{j}^{T} & \equiv \overline{\rho u_{j}^{\prime \prime} h^{\prime \prime}} \\
\tau_{i j}^{T} & \equiv-\overline{\rho u_{i}^{\prime \prime} u_{j}^{\prime \prime}} \\
\bar{\rho} k & \equiv \frac{1}{2} \overline{\rho u_{i}^{\prime \prime} u_{i}^{\prime \prime}}
\end{aligned}
$$

\section{II.A. Model Calibration}

Turbulence models are approximations used to mimic the mean behavior of the actual physical processes, and as such usually involve closure coefficients that must be calibrated through comparison of results with known or expected behavior. This is usually done with a series of fundamental flows that isolate specific terms. Each turbulence model may have been calibrated using a different procedure, a different set of experimental data, or a different metric for "optimizing" the results. Unfortunately, the details of how a model has been calibrated are not always fully disclosed.

To illustrate how models are calibrated, consider the form of a typical $k-\epsilon$ eddy viscosity model which has been routinely applied to free shear flows.

$$
\begin{gathered}
\tau_{i j}^{T} \equiv-\overline{\rho u_{i}^{\prime \prime} u_{i}^{\prime \prime}}=2 \mu_{T} \widetilde{S}_{i j}^{D}-\frac{2}{3} \bar{\rho} k \delta_{i j} \\
\mu_{T}=C_{\mu} f_{\mu} \bar{\rho} k^{2} / \epsilon \\
\frac{\partial(\bar{\rho} k)}{\partial t}+\frac{\partial\left(\bar{\rho} \widetilde{u}_{j} k\right)}{\partial x_{j}}=\frac{\partial}{\partial x_{j}}\left[\left(\mu_{L}+\frac{\mu_{T}}{\sigma_{k}}\right) \frac{\partial k}{\partial x_{j}}\right]+\mathcal{P}-\rho \epsilon+L_{k} \\
\frac{\partial(\bar{\rho} \epsilon)}{\partial t}+\frac{\partial\left(\bar{\rho} \widetilde{u}_{j} \epsilon\right)}{\partial x_{j}}=\frac{\partial}{\partial x_{j}}\left[\left(\mu_{L}+\frac{\mu_{T}}{\sigma_{\epsilon}}\right) \frac{\partial \epsilon}{\partial x_{j}}\right]+C_{\epsilon 1} f_{1} \frac{\epsilon}{k} \mathcal{P}-C_{\epsilon 2} f_{2} \frac{\rho \epsilon^{2}}{k}+L_{\epsilon} \\
\mathcal{P} \equiv \tau_{i j}^{T} \frac{\partial \widetilde{u}_{i}}{\partial x_{j}}
\end{gathered}
$$

In these equations, $f_{\mu}, f_{1}$, and $f_{2}$ represent damping functions and $L_{k}$ and $L_{\epsilon}$ represent near-wall terms, the details of which are not important for this discussion. The eddy viscosity coefficient $C_{\mu}$ is often calibrated under the assumption of thin shear flows where the production of turbulent kinetic energy is nearly in balance with the turbulent dissipation rate $(\mathcal{P} \approx \bar{\rho} \epsilon)$ together with the experimental data of Townsend ${ }^{74}$ which indicates that the ratio of shear stress to turbulent kinetic energy for a wide range of flows has nearly the same value of 0.3 . The $C_{\epsilon 2}$ coefficient multiplying the dissipation term in the $\epsilon$-transport equation is determined by analyzing the decay of isotropic homogeneous turbulence. The $C_{\epsilon 1}$ coefficient multiplying the production term in the $\epsilon$-transport equation is obtained by applying the model to homogeneous turbulent shear flow. The diffusion coefficient $\sigma_{\epsilon}$ that also appears in the $\epsilon$-transport equation is calibrated by analyzing the log-layer region of the turbulent boundary layer. The diffusion coefficient $\sigma_{k}$ the $k$-transport equation is typically set to 1.0 .

An $\epsilon$-based Reynolds stress model requires the calibration of a number of coefficients in the model for the pressure-strain correlation tensor. The following quasi-linear form is commonly used.

$$
\begin{aligned}
\frac{\Pi_{i j}^{D}}{\bar{\rho} \epsilon}=-\left(C_{1}^{0}+C_{1}^{1} \frac{\mathcal{P}}{\bar{\rho} \epsilon}\right) b_{i j}+C_{2} \frac{k}{\epsilon} \widetilde{S}_{i j}^{D} & +C_{3} \frac{k}{\epsilon}\left[b_{i k} \widetilde{S}_{k j}+\widetilde{S}_{i k} b_{k j}-\frac{2}{3} b_{m n} \widetilde{S}_{m n} \delta_{i j}\right] \\
& -C_{4} \frac{k}{\epsilon}\left[b_{i k} \widetilde{R}_{k j}-\widetilde{R}_{i k} b_{k j}\right]
\end{aligned}
$$

The "slow" pressure-strain coefficient $C_{1}^{0}$ is calibrated from the decay of anisotropic homogeneous turbulence. The remaining pressure-strain coefficients $C_{1}^{1}, C_{3}$, and $C_{4}$ are optimized to provide agreement with homogeneous shear flow and rotating shear flow, while $C_{2}$ is determined from rapid distortion theory for homogeneously strained turbulence that is initially isotropic. 
One shortcoming of any such calibration procedure is that it is assumed that these coefficients will retain the same values for all types of flows. Bradshaw ${ }^{75}$ notes that the coefficients used in the modeling terms will only assume constant values in the unlikely event that the modeled term exactly correlates with the physical term being modeled. More plausibly these coefficients will be different for different flows, and may vary from point to point within the same flow. He suggests that these coefficients may be improved by making them empirical functions of the local flow parameters such as the ratio of production to dissipation or a normalized measure of the flow invariants.

\section{II.B. Differences Between Planar and Axisymmetric Flows}

Free shear flows are composed of a variety of three-dimensional turbulent structures. However, the organization of those structures is related to the dominant instability modes, which differ between mixing layers, jets, and wakes. These differences can be seen in experimental flow visualization and in solutions from largeeddy simulation. This change in the way that the turbulence is organized presents a particular challenge for RANS-based methods which model statistical averages rather than structure. It is among the reasons why most turbulence models cannot accurately predict both mixing layers and jets using the same set of coefficients.

Table 2 compares RANS model predictions for spread rates in the self-similar regions of different types of free shear flows. From these results one can see that every turbulence model predicts each type of flow with varying degrees of success. For example, the Spalart-Allmaras ${ }^{76}$ model does a poor job of predicting jet flows, but excels in the wake flows for which it was specifically calibrated. In the case of the widely used $k-\epsilon$ model of Launder and Sharma, ${ }^{77}$ the spread rate is in agreement with the experimental data for the mixing layer, plane jet and radial jet, but is too fast for the round jet.

Table 2: Free shear flow spread rates predicted by RANS models.

\begin{tabular}{|c|c|c|c|c|c|c|}
\hline & & Mixing Layer & Plane Jet & Round Jet & Radial Jet & Wake \\
\hline & Experiment $^{7,78-81}$ & $0.103-0.120$ & $0.100-0.110$ & $0.086-0.096$ & $0.096-0.110$ & $0.320-0.400$ \\
\hline$k-\omega$ & Wilcox $^{82 *}$ & 0.096 & 0.108 & 0.094 & 0.099 & 0.326 \\
\hline$k-\omega$ & Wilcox ${ }^{83 *}$ & 0.105 & 0.101 & 0.088 & 0.099 & 0.339 \\
\hline$k-\omega$ & Wilcox $^{84^{*}}$ & 0.141 & 0.135 & 0.369 & 0.317 & 0.496 \\
\hline $\mathrm{SST}$ & Menter $^{85 \dagger}$ & 0.100 & 0.112 & 0.127 & - & 0.257 \\
\hline$k-\epsilon$ & Launder \& Sharma $^{77^{*}}$ & 0.098 & 0.109 & 0.120 & 0.094 & 0.256 \\
\hline & with Pope P6* $^{8 *}$ & 0.098 & 0.109 & 0.086 & 0.040 & 0.256 \\
\hline$k-\zeta$ & Robinson, et al. ${ }^{87 *}$ & 0.112 & 0.115 & 0.091 & 0.097 & 0.313 \\
\hline$k-\tau$ & Speziale, et al. ${ }^{88^{*}}$ & 0.082 & 0.089 & 0.102 & 0.073 & 0.221 \\
\hline SA & Spalart \& Allmaras ${ }^{76^{*}}$ & 0.109 & 0.157 & 0.248 & 0.166 & 0.341 \\
\hline
\end{tabular}

${ }^{*}$ Results reported by Wilcox. ${ }^{82}$

${ }^{\dagger}$ Results reported by Bardina, et al. ${ }^{89}$

Pope $^{86}$ developed an additional parameter to improve $k-\epsilon$ model round jet predictions based on the concept of vortex stretching. He theorized that the stretching of turbulent vortex tubes by the mean flow has a significant influence on the rate of turbulent dissipation. As these vortices are stretched, there is a transfer of energy from the large eddies to the smaller ones. Since the small eddies control the dissipation, the dissipation rate $\epsilon$ must increase. To achieve this effect, Pope proposed a model correction to the $\epsilon$-transport equation that reduces the effective dissipation of $\epsilon$.

$$
C_{\epsilon 2} f_{2} \frac{\bar{\rho} \epsilon^{2}}{k} \rightarrow\left(C_{\epsilon 2} f_{2}-C_{\epsilon 3} f_{3} \chi_{p}\right) \frac{\bar{\rho} \epsilon^{2}}{k}
$$

$\chi_{p}$ is a nondimensional measure of the vortex stretching.

$$
\chi_{p} \equiv\left(\frac{k}{\epsilon}\right)^{3} \widetilde{R}_{i j} \widetilde{R}_{j k} \widetilde{S}_{k i}
$$

Similar terms have been incorporated into other turbulence models. The Wilcox ${ }^{82,83} k-\omega$ and Robinson, et al. ${ }^{87} k-\zeta$ models include a term of this type into their baseline models. As shown in table 2 , these models 
do reasonably well for each type of free shear flow. Depending upon the behavior of the baseline turbulence model, this correction can adversely affect the prediction of other three-dimensional flows where $\chi_{p} \neq 0$. Such is the case with the $k-\epsilon$ model, where the spread rate for the radial jet is significantly reduced.

For subsonic round jets, baseline $k-\epsilon$ models underpredict the growth of the initial shear layer, resulting in potential core lengths that are too long. Near the end of the potential core and farther downstream, the shear layer growth rate is too high relative to data. Activation of the vortex stretching correction with these models will reduce the level of turbulence and slow the shear layer growth rate throughout the flowfield. While this action may bring the self-similar mixing rate in line with experimental data, it will have the adverse effect of yielding potential core lengths that are even longer than with the baseline model.

Birch $^{90,91}$ has questioned the physical basis for this correction, noting that experimental results have shown lateral divergence actually increases the turbulent shear stress. Regardless, this method is still sometimes used because it tends to shift the self-similar RANS predictions in the right direction for round jets.

In algebraic Reynolds stress modeling, the turbulence anisotropy tensor can be related to ten basis tensors and six invariants formed from combinations of the mean strain and rotation rate tensors. ${ }^{92}$ It is interesting to note that the expression $\widetilde{R}_{i j} \widetilde{R}_{j k} \widetilde{S}_{k i}$ used in Pope's vortex stretching parameter in Eq. (30) matches one of these six invariants: $\left\{\widetilde{\boldsymbol{S}}^{2}\right\},\left\{\widetilde{\boldsymbol{R}}^{2}\right\},\left\{\widetilde{\boldsymbol{S}}^{3}\right\},\left\{\widetilde{\boldsymbol{S}} \widetilde{\boldsymbol{R}}^{2}\right\},\left\{\widetilde{\boldsymbol{S}}^{2} \widetilde{\boldsymbol{R}}^{2}\right\},\left\{\widetilde{\boldsymbol{S}}^{2} \widetilde{\boldsymbol{R}}^{2} \widetilde{\boldsymbol{S}} \widetilde{\boldsymbol{R}}\right\}$. This lends some support to the previously described notion that perhaps the closure coefficients should not be constants, but functions of the flow invariants.

Considering the nature of turbulent jet flows, it is surprising that RANS models do as well as they do. The initial plane (or axisymmetric) jet region is similar to planar (or annular) mixing layers. Near the end of the potential core, these shear layers interact with each other and merge together. Farther downstream the plume resembles a single shear layer. In each region, the composition of turbulent structures differ, and one might expect each region to require its own set of coefficients. Defining where and how those coefficients should transition would most likely limit the applicability of any such model to other types of flow.

Over a decade ago, Birch ${ }^{91}$ described the dichotomy between Reynolds-averaged methods and structurebased methods such as large-eddy simulation. From the coherent structure viewpoint, shear layer growth is likely sensitive to the detailed structure of the large eddies. Even though a self-similar region might be reached, the turbulent mixing could continue to depend upon the initial turbulent structure and will never truly be the same. From the Reynolds-averaged perspective, initial conditions might persist for some distance downstream, but an asymptotic state is eventually reached that is unique for each flow geometry (mixing layer, jet, etc.).

Of these two perspectives, it seems more likely that structure-based methods should provide more accurate simulations. Large-eddy simulation methods resolve the most important turbulent scales through the use of the unsteady Navier-Stokes equations. RANS models are instead based upon a time (or ensemble) average of those equations, which as a simplified subset of the full equations will surely contain less information. If structure does play such a key role in these types of turbulent flows, then it will be difficult for RANS models to accurately replicate such physics even in an averaged sense.

\section{II.C. The Developing Region}

The types of homogeneous and isotropic benchmark cases used to calibrate the coefficients in RANS-based turbulence models are rather idealized and might, at best, resemble that observed in the self-similar region that exists far downstream. The developing region is frequently ignored when assessing the performance of RANS models, as the spreading rate in the self-similar region is usually emphasized (i.e., table 2). For propulsion applications, however, the primary region of interest is often in the near field. As an example, the mixing that occurs in scramjets and internal mixer nozzles is almost always done in the minimum length possible. Also, most of the noise in a subsonic jet is generated within the approximate length of the potential core.

In the development region, the turbulence is in a non-equilibrium state and could even be transitioning from laminar to turbulent. The mean and turbulence profiles vary spatially but, unlike the downstream region, are not self-similar. The development region can also be influenced by the upstream flow, including inflow profiles, boundary layer state (laminar or turbulent), and freestream turbulence levels. It is unclear whether RANS methods can be made sensitive to all of the factors influencing the development region, or whether one should use LES instead. 


\section{II.D. Compressibility}

In RANS simulations, the most common means for including compressibility effects on turbulence is through the inclusion of explicit dilatation terms that appear in the transport equations for the Reynolds normal stresses (and subsequently the turbulent kinetic energy). These terms, which include the dilatation dissipation $^{93}\left(\epsilon_{d}\right)$ and the pressure dilatation ${ }^{94}\left(\Pi^{d i l}\right)$, have an additional dissipative effect on the growth of turbulence. As shown in figure 9 from reference 6, these terms provide varying degrees of growth rate reduction in compressible mixing layers. Both of the dilatation models shown are functions of the turbulent

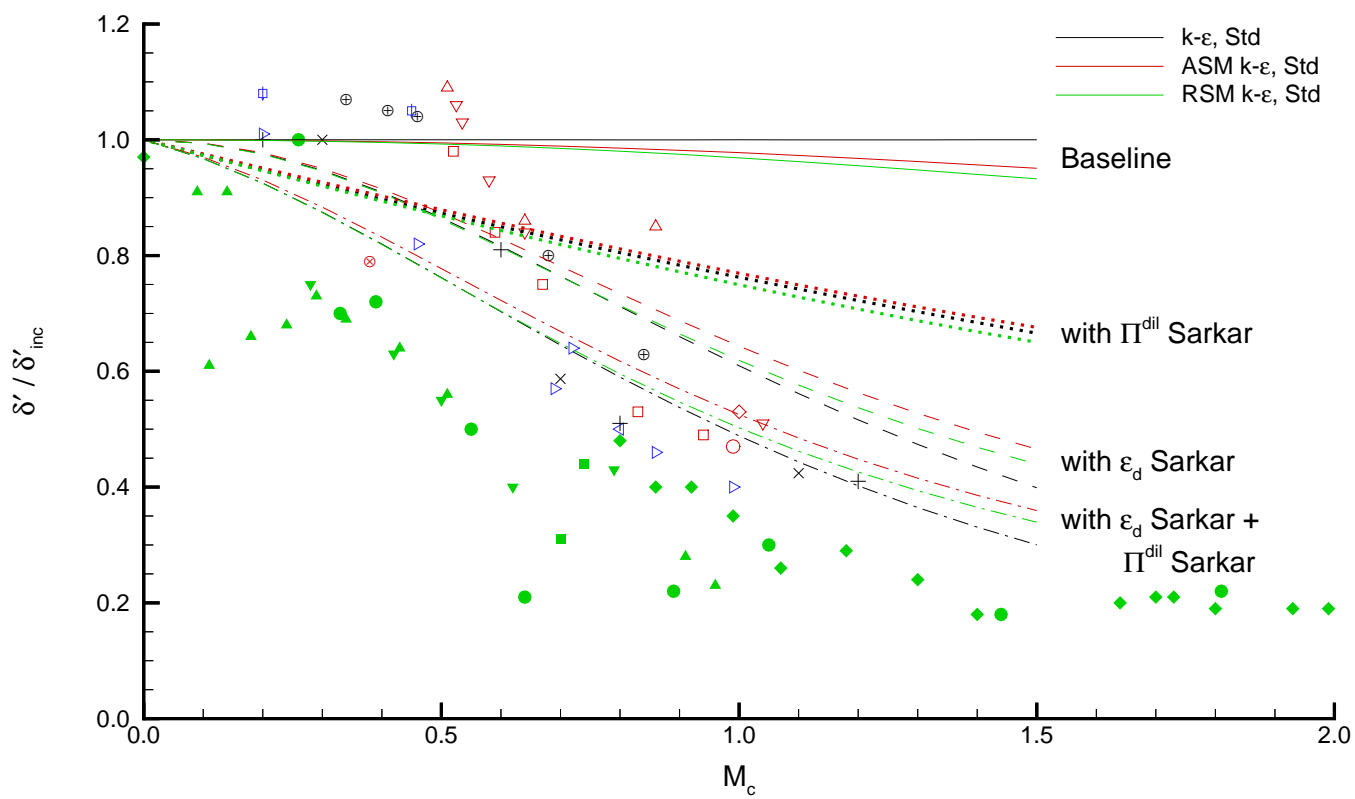

Figure 9: RANS predictions of compressible mixing layer growth rate from reference 6 .

Mach number and display sensitivity to compressibility even at low $M_{c}$. Therefore, the resulting change in growth rate is more gradual than indicated by the data. In addition, these methods do not significantly alter the Reynolds stress anisotropy, because the normal stresses are all reduced in an isotropic manner.

Dembowski and Georgiadis ${ }^{95}$ investigated the ability of typical RANS models to predict a Mach 2 round jet at different operating temperatures. ${ }^{96}$ The results, in figure 10, show that the baseline SST model mixes much faster than indicated by the experimental data. This is consistent with the observation that standard models predict shear layers that grow too quickly because the models are not sensitive to compressibility effects. Use of a dilatation dissipation correction slows the growth of turbulence, but results in a potential core length that is too long. Farther downstream the decay rate is still slightly over-predicted for the unheated case, but this trend reverses for the higher temperatures.

Results from direct numerical simulation (DNS) of homogeneous shear flows,${ }^{97}$ temporal mixing layers, ${ }^{55}$ and annular mixing layers ${ }^{98}$ indicate that the contributions of dilatation dissipation and pressure dilatation are both insignificant. Instead, reduced pressure fluctuations (i.e., reduced communication) across the compressible shear layer alter the pressure-strain correlation tensor that appears in the Reynolds stress transport equation. This tensor redistributes the turbulence energy between the streamwise, transverse, and spanwise terms. While each of the Reynolds stresses are reduced with increasing compressibility, the streamwise normal stress is less affected and leads to an increase in the turbulence anisotropy. However, for temporal mixing layers, the DNS of Pantano and Sarkar ${ }^{34}$ suggests that the degree to which turbulence anisotropy changes can depend upon the extent and duration of the simulation.

Since compressibility effects appear to be linked to changes in the pressure-strain correlation tensor, accurate physical modeling of compressible mixing layers requires (at a minimum) the use of algebraic or differential Reynolds stress models. Formulation of a truly compressible model for the pressure-strain correlation is a formidable challenge. The more common approach has been to use existing pressure-strain models and apply damping functions ${ }^{34,99-101}$ to modify some or all of the pressure-strain model coefficients. These damping functions depend upon some measure of compressibility, such as the convective $\left(M_{c}\right)$, turbulent $\left(M_{t}=\sqrt{2 k} / a\right)$, or gradient $\left(M_{g}=|S| l / a\right)$ Mach number. Of these, the gradient Mach number appears to 


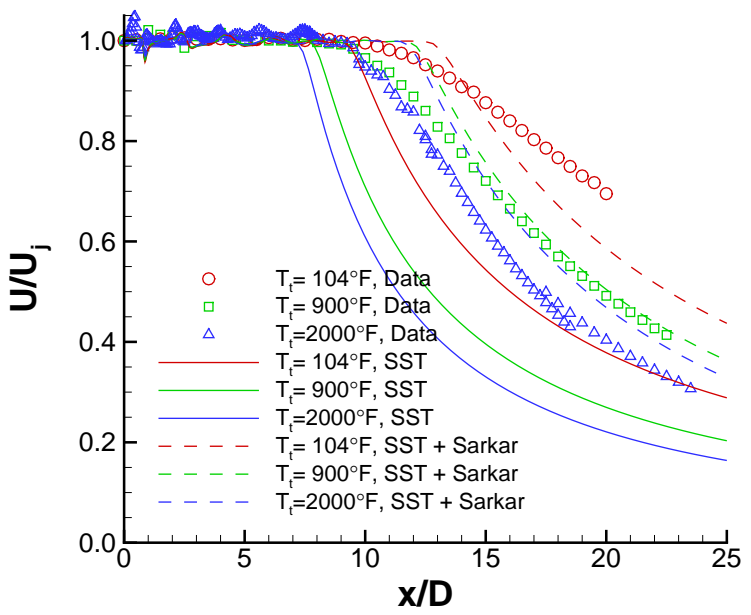

(a) Centerline axial velocity

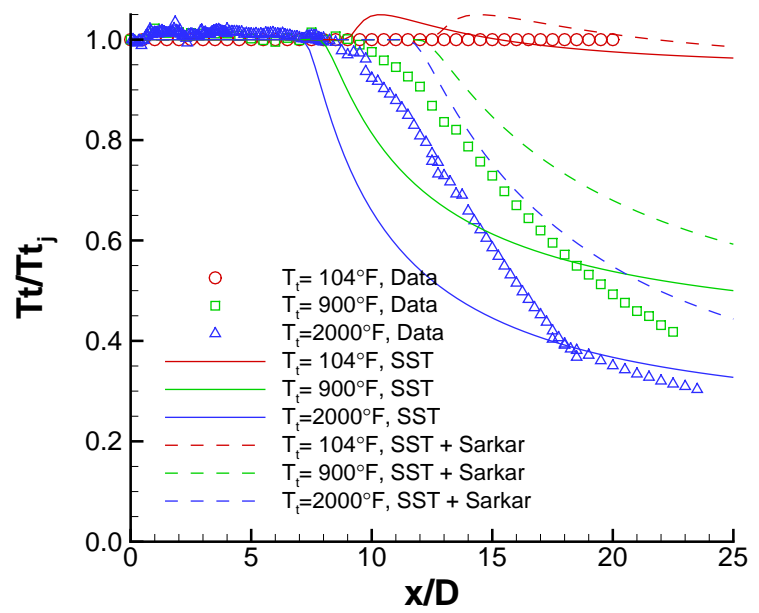

(b) Centerline stagnation temperature

Figure 10: Effect of temperature on jet predictions from reference 95.

be the most appropriate choice since the convective Mach number is a global parameter and the turbulent Mach number can be large in both high-speed mixing layers and boundary layers.

Gomez and Girimaji ${ }^{102}$ suggest that modeling of the pressure strain correlation tensor can be split into three distinct regimes. At low gradient Mach number, a standard incompressible pressure-strain correlation model may be used. At intermediate $M_{g}$, the pressure and inertial terms are of the same magnitude such that the pressure-strain tensor approximately balances production. At high $M_{g}$, compressibility severely limits pressure communication and the contribution of the pressure-strain tensor becomes negligible. The transition between regimes is accomplished by a blending function.

Once again, it is interesting to note that the parameter used to affect a change in the turbulence model predictions can be related to the flow invariants. In this case, the gradient Mach number can be related to $\left\{\boldsymbol{S}^{2}\right\}$. Thus, procedures that sensitize the model coefficients to $M_{g}$ are, in effect, sensitizing them to one of the local flow invariants.

\section{II.E. Thermal and Mass Effects}

Propulsion flows generally involve gaseous species that are the products of combustion at elevated pressure and temperature. As the exhaust expands out the nozzle, it undergoes a significant temperature change. Density and thermal gradients in the jet add another level of complication to modeling.

The review by Georgiadis and DeBonis ${ }^{103}$ includes solutions of various RANS models for unheated and heated incompressible round jets. Some of the models were specifically tuned for jet flows and/or temperature effects. For an unheated jet, baseline two-equation turbulence models generally predict potential core lengths that are too long and downstream mixing rates that are too high. While the peak value of turbulent kinetic energy along the centerline is well represented, it does not diffuse to the centerline as quickly as the data indicates. For the heated jet, the baseline models again predict a potential core that is too long. They also predict a centerline turbulence profile that is similar to the unheated case, albeit shifted slightly upstream, and fail to predict the higher peak turbulence level. Through a modification of the turbulent viscosity, the temperature corrected model mixes more quickly than the standard model, providing better centerline velocity decay. However, the turbulent kinetic energy field dissipates much too quickly.

In RANS simulations, Reynolds' analogy between momentum and energy is typically used with an assumed constant turbulent Prandtl number. A value of 0.89 is common for boundary layers, while a value as low as 0.5 is more appropriate in free shear flows. Dembowski and Georgiadis ${ }^{95}$ investigated the effect of turbulent Prandtl number on Mach 2 hot round jet simulations. As shown in figure 11, higher values of $\operatorname{Pr}_{T}$ have little effect on the velocity field, but slightly reduce the thermal decay.

Turbulent Prandtl and Schmidt number variations also affect the mixing and combustion that occurs in reacting mixing layers. The simulation results ${ }^{104}$ for a supersonic reacting mixing layer ${ }^{105}$ are shown in 


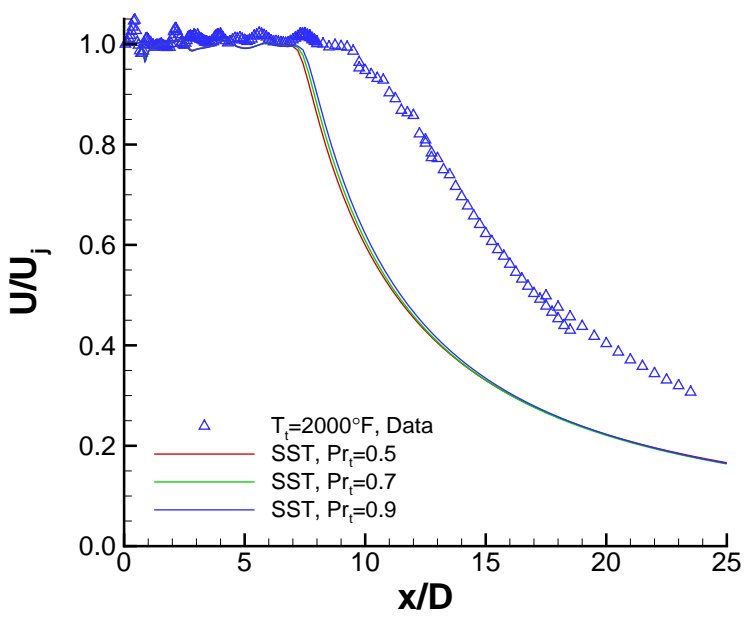

(a) Centerline axial velocity

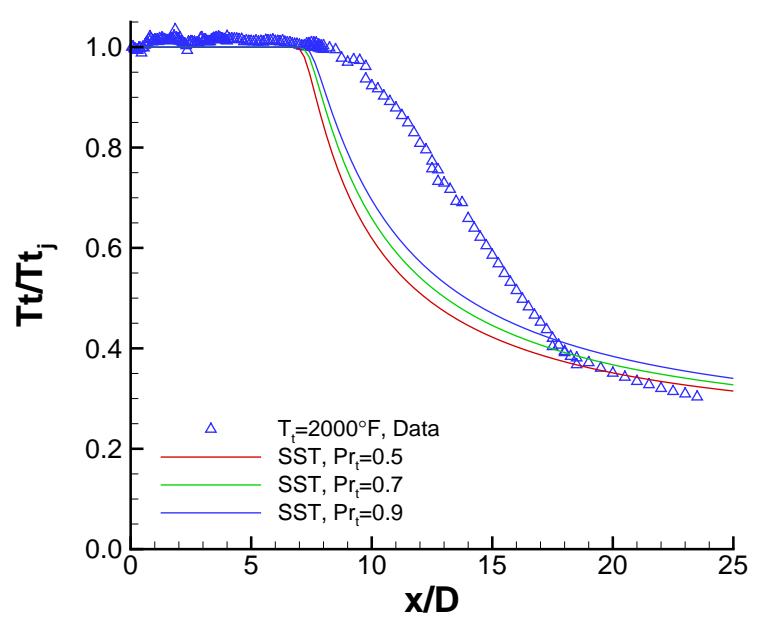

(b) Centerline stagnation temperature

Figure 11: Effect of turbulent Prandtl number on hot jet predictions from reference 95.

figure 12. For this case, the ignition point moves slightly upstream with increasing $P r_{T}$. The results show a much greater sensitivity to $S c_{T}$, which influences the species mass diffusivity much in the same way that the turbulent Prandtl number influences the thermal diffusivity. As the turbulent Schmidt number is reduced, the species diffuse more quickly creating a more favorable mix of reactants for combustion to occur sooner.

Unfortunately, the optimal turbulent Prandtl and Schmidt numbers for one case are not optimal for others. In general the values will vary throughout the flowfield. Some work has been done in the area of variable turbulent Prandtl and Schmidt number modeling. ${ }^{106-109}$ Most of these models relate the thermal (or mass) diffusivity to the scalar variance of temperature (or species mass fractions), then solve modeled transport equations for that variance and its dissipation rate. For combusting flows, development of these models is complicated by the consideration of turbulent-chemistry interaction effects, which are sometimes included through probability density function (PDF) formulations. A major hindrance to the development of variable $\operatorname{Pr}_{T}$ and $S c_{T}$ models is a lack of experimental data to calibrate and validate the thermal and mass diffusivities, and underlying scalar transport equations. While these methods are promising, they are not yet mature enough for routine use.

\section{LES/DNS Modeling Issues}

Large-eddy simulation and direct numerical simulation hold great promise for improving the predictive capability of jets and mixing layers. These scale-resolving methods directly compute the key turbulent structures. DNS directly computes the full turbulent spectrum all the way down to the Kolmogorov scales. LES uses a spatial filtering procedure to separate the unsteadiness into large, resolved scales and small, unresolved scales. LES directly computes the resolved scales and approximates the effect of the unresolved scales using a sub-grid model. Because of the very fine grids required to resolve all scales, DNS for most flows at realistic Reynolds numbers is impractical using current computer technology. LES significantly relaxes the computational requirements by only resolving the large scales and has been used extensively for jets. The focus of the discussion here will be on LES techniques.

In LES, a spatial filter $G$ with a filter width $\Delta$ is used to separate the resolved and unresolved scales. For example,

$$
\bar{u}_{i} \equiv \int_{-\infty}^{\infty} G(x-\xi) u_{i}(\xi) d \xi
$$

The overbar represents the resolved, filtered, or large-scale portion of the variable. For compressible flows a 


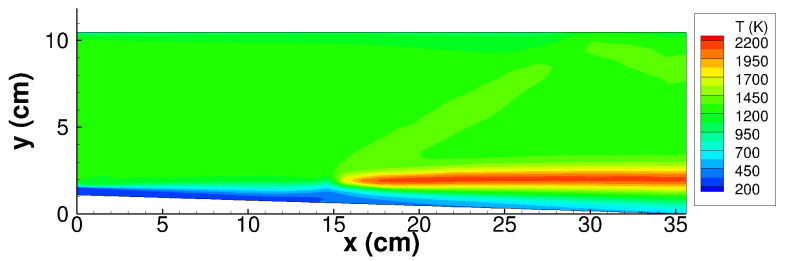

(a) $P r_{t}=0.5, S c_{t}=0.7$

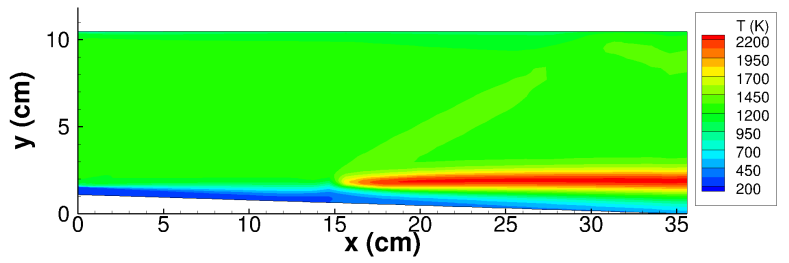

(c) $P r_{t}=0.7, S c_{t}=0.7$

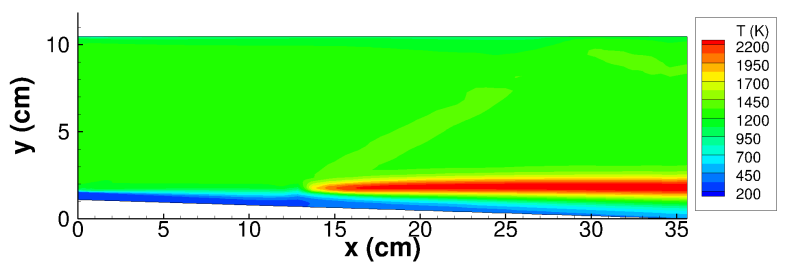

(e) $P r_{t}=0.9, S c_{t}=0.7$

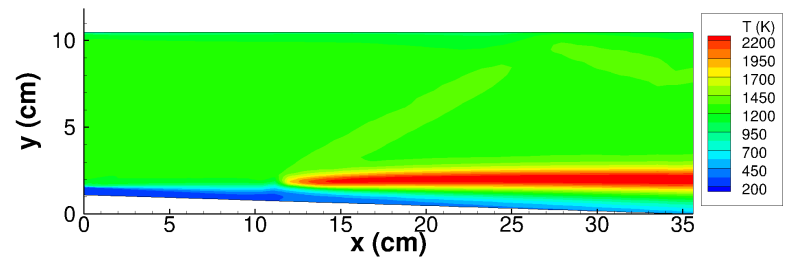

(b) $P r_{t}=0.7, S c_{t}=0.5$

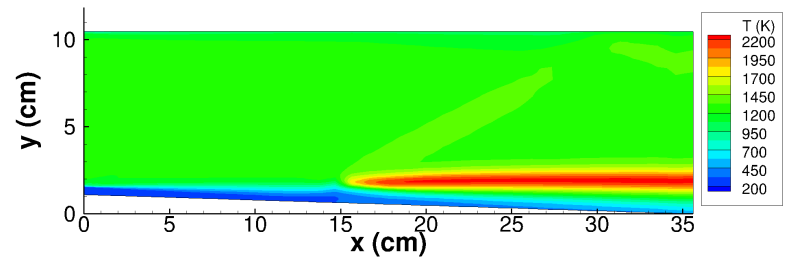

(d) $P r_{t}=0.7, S c_{t}=0.7$

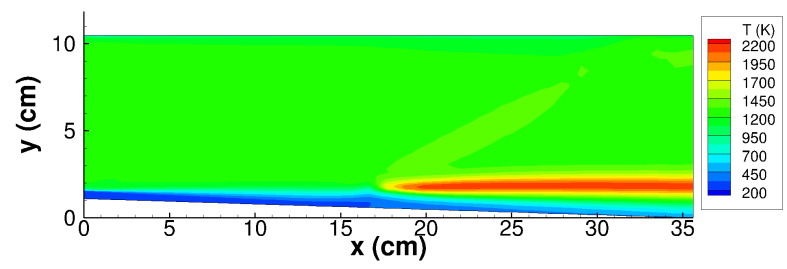

(f) $P r_{t}=0.7, S c_{t}=0.9$

Figure 12: Effect of turbulent Prandtl number (a,c,e) and Schmidt number (b,d,f) on combustion, from reference 104 . 
density-weighted filter is often used instead.

$$
\widetilde{u} \equiv \overline{\rho u} / \bar{\rho}
$$

Applying the filter to the Navier-Stokes equations yields the following forms of the continuity, momentum, and energy equations

$$
\begin{gathered}
\frac{\partial \bar{\rho}}{\partial t}+\frac{\partial \bar{\rho} \widetilde{u}_{j}}{\partial x_{j}}=0 \\
\frac{\partial \bar{\rho} \widetilde{u}_{i}}{\partial t}+\frac{\partial}{\partial x_{j}}\left[\bar{\rho} \widetilde{u}_{i} \widetilde{u}_{j}+\bar{P} \delta_{i j}-\left(\bar{\tau}_{i j}+\tau_{i j}^{s g s}\right)\right]=0 \\
\frac{\partial}{\partial t}\left[\bar{\rho}\left(\widetilde{e}+\frac{1}{2} \widetilde{u}_{i} \widetilde{u}_{i}+k^{s g s}\right)\right]+\frac{\partial}{\partial x_{j}}\left[\bar{\rho} \widetilde{u}_{j}\left(\widetilde{h}+\frac{1}{2} \widetilde{u}_{i} \widetilde{u}_{i}+k^{s g s}\right)-\widetilde{u}_{i}\left(\bar{\tau}_{i j}+\tau_{i j}^{s g s}\right)+\left(\bar{q}_{j}+q_{j}^{s g s}\right)\right]=0
\end{gathered}
$$

where the sub-grid scale (SGS) heat flux, stress, and kinetic energy are defined as:

$$
\begin{aligned}
q_{j}^{s g s} & \equiv \bar{\rho}\left(\widetilde{u_{j} h}-\widetilde{u}_{j} \widetilde{h}\right) \\
\tau_{i j}^{s g s} & \equiv-\bar{\rho}\left(\widetilde{u_{i} u_{j}}-\widetilde{u}_{i} \widetilde{u}_{j}\right) \\
\bar{\rho} k^{s g s} & \equiv \frac{1}{2} \bar{\rho}\left(\widetilde{u_{i} u_{i}}-\widetilde{u}_{i} \widetilde{u}_{i}\right)
\end{aligned}
$$

These sub-grid scale terms must be modeled. However, unlike in the RANS equations where all of the turbulent effects were replaced by a turbulence model, the SGS terms only represent that portion of the turbulent motion that is at too small of a scale to be resolved by the computational scheme and mesh.

A large-eddy simulation is a complex interaction between computational grid, numerical scheme, boundary conditions, sub-grid model, and filter. The key to the success of LES is properly resolving the large-scale turbulent structures that contain the majority of the turbulent energy in the flow. Maximizing the resolution of the simulation minimizes the effect of the sub-grid model. Because LES directly computes the large-scale structures, it better represents the turbulent flow physics and is not subject to the types of modeling errors associated with RANS simulations of jets and mixing layers. The effects of compressibility on turbulence and mixing will be largely captured directly, with only minimal errors occurring due to the sub-grid model. The primary LES issue specific to free shear flows is the proper resolution of the developing region. This requires sophisticated boundary conditions to simulate the initial turbulent inflow and high-resolution schemes together with high-quality grids to capture the small but important turbulent structures.

A brief summary of the key LES issues is presented below. Georgiadis and DeBonis ${ }^{103}$ include a more complete review of the subject.

\section{III.A. Numerical Scheme}

The numerical scheme used in LES/DNS calculations can have a significant effect on the computed results. In order to resolve the evolution of the turbulent structures without damping them, schemes that maximize wave resolution and minimize numerical dissipation are desirable. For this reason, high-order explicit finitedifferences are often used to maximize the order of accuracy of the scheme. Dispersion relation preserving (DRP) schemes further optimize the discretization stencil coefficients to minimize the dispersion error over a limited range of scales. ${ }^{110,111}$ Compact schemes are also popular and increase the resolution for a given stencil size by implicitly computing the derivatives. ${ }^{112}$ Very low dissipation methods based on kinetic energy conserving schemes have shown significant promise for low-order unstructured grid codes. ${ }^{113}$

Central difference formulations are desirable for these types of simulations because they lack inherent dissipation. However, they will be numerically unstable unless some form of artificial dissipation is added. This is frequently done through the application of a filter function which is designed to work with a particular scheme. Upwind schemes are naturally more dissipative than central difference methods and will damp the turbulent structures. Use of higher-order upwinding is usually necessary to allow the growth and evolution of the turbulence. Note that regardless of the scheme used, the order of accuracy only indicates how the error will be reduced with grid refinement and does not indicate the magnitude of the error for a given grid size.

Compressibility introduces another complexity for large-eddy simulation, namely how to handle shock waves that are present in the flow. In central difference solvers, a trigger based on the pressure gradient is 
often used to activate localized artificial dissipation or to switch to a blended central/upwind method in the region near a shock. Pure upwind-based methods tend to be more stable near shocks due to their higher levels of inherent dissipation. Total variation diminishing (TVD) limiters may also be used to reduce the pressure fluctuations on either side of the shock. Applying these fixes near shocks is a greater challenge in LES simulations than in RANS because any additional damping will act to weaken the resolved turbulent structures. Therefore, care must be taken to only apply enough dissipation to keep the scheme stable in the shock region. The localized artificial diffusivity (LAD) technique ${ }^{114}$ provides shock stability without affecting turbulent structures by adding artificial dissipation through the bulk viscosity term, leaving the shear viscosity and the stress tensor unaffected.

\section{III.B. Computational Mesh}

Numerical schemes are sensitive to the computational mesh used. Each scheme has its own requirements for the number of points to resolve a particular wave number, grid skewness, and rate of grid stretching. Higher-order methods used in LES/DNS often have wide discretization stencils that introduce additional grid requirements and make the treatment of complex geometry challenging. Isotropic grids are best for capturing the rotating nature of the turbulent structures as they convect downstream.

For LES/DNS, the range of scales that must be resolved is Reynolds number dependent. As the Reynolds number increases, the Kolmogorov length scale, which represents the smallest eddies, will decrease. While LES does not resolve all the way down to the Kolmogorov scale, the range of "important" scales will likewise grow. For higher Reynolds number flows, this means that a finer grid will be needed to resolve a similar percentage of the overall scales or else the sub-grid model will be relied upon to model their effect.

In a time accurate simulation such as LES, the size of the step used to advance the solution in time is directly proportional to the smallest grid spacing in the domain through the Courant-Friedrichs-Lewy (CFL) stability condition for the numerical scheme used. Increasing the grid resolution not only increases the computational time per iteration to solve the larger grid, but it also increases the number time steps necessary to achieve the same total simulation time. It is important to note that the total simulation time needed is determined by the size and frequency of the large-scale structures, and remains fixed for a given flow.

Wall bounded flows likewise present a challenge for LES, because of the range of scales involved. This also becomes important for free shear flow applications, because the state of the upstream boundary layers plays an important role in the development region of the shear layer. Even if the unsteady flow entering the free shear region is prescribed, the downstream grid must be fine enough to resolve the incoming turbulent eddies and give them a chance to grow. Otherwise the numerical dissipation will dampen if not destroy the turbulent fluctuations.

As an example, consider the case of an axisymmetric jet exiting from a convergent nozzle from reference 115. The flow conditions for this subsonic unheated jet are very similar to setpoint 7 in table 1 . Figure 13 , which compares the centerline velocity decay, reveals a potential core that is a little too short and a decay rate that is too fast in the region near the end of the core, but then a mixing rate that seems to improve farther downstream. Examination of the root mean square (RMS) velocities downstream of the nozzle lip (figure 14) reveal initial values that are much too high, but then provide better agreement farther downstream. Because the grid near the nozzle exit is not fine enough to capture the small-scale turbulence from the boundary layers, the LES simulation sees this as a more laminar-like jet. The elevated RMS velocities downstream of the nozzle lip (figure 15) are characteristic of laminar to turbulent transition. This laminar-like inflow results in the shorter potential core, but as more of the energy-containing eddies are resolved farther downstream the rate of centerline velocity decay improves.

\section{III.C. Boundary Conditions}

Boundary conditions present a particular challenge for LES/DNS. Rarely are the unsteady inflow conditions known to the level of fidelity needed, and computing the entire unsteady domain is not usually feasible. Instead several techniques have been developed to provide approximate inflow conditions. Synthetic turbulence methods impose an unsteady velocity profile based on a specified mean flow and Reynolds stress profile. The specified profiles may come from experimental data or a RANS simulation that is run a priori or in a coupled hybrid RANS/LES fashion. Various techniques have been developed to compute the fluctuating quantities 


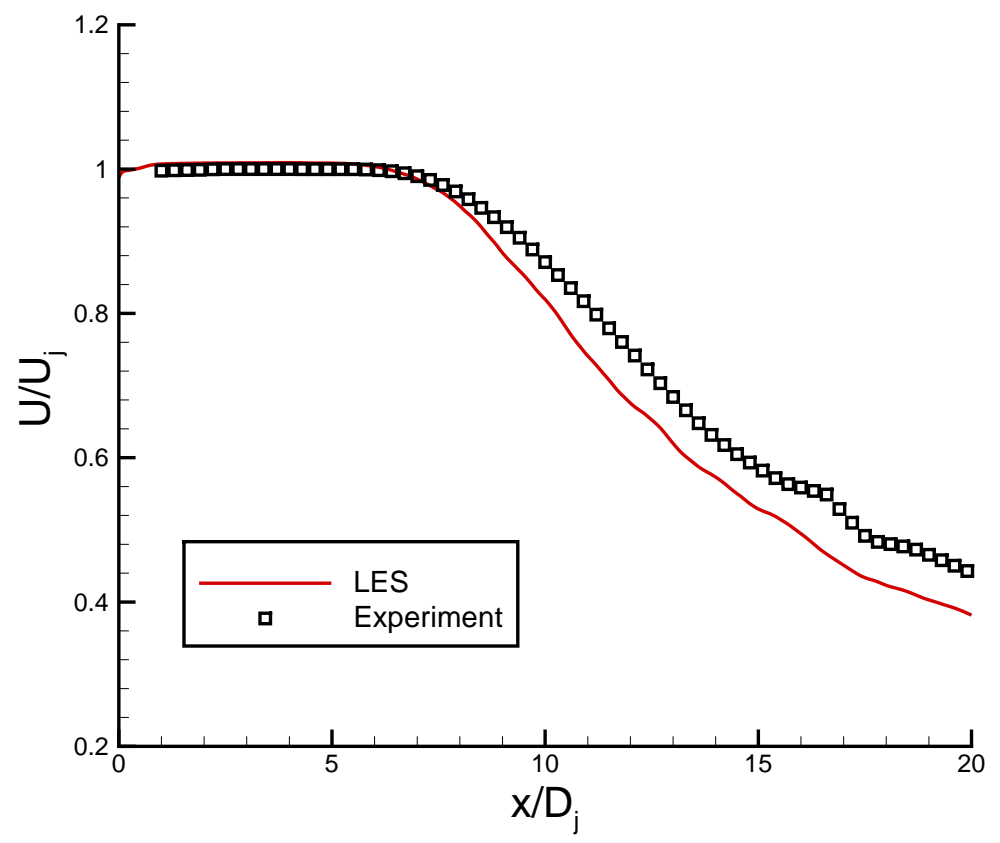

Figure 13: LES axisymmetric jet centerline velocity from reference 115.

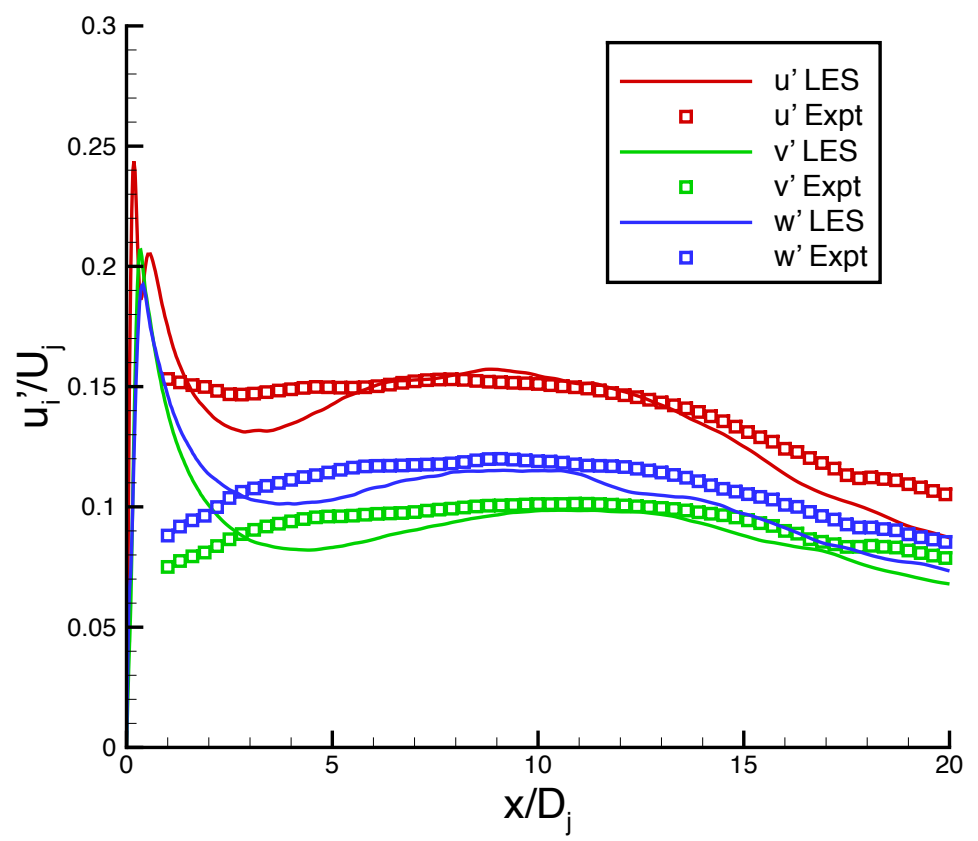

Figure 14: LES axisymmetric jet lip line turbulence from reference 115. 


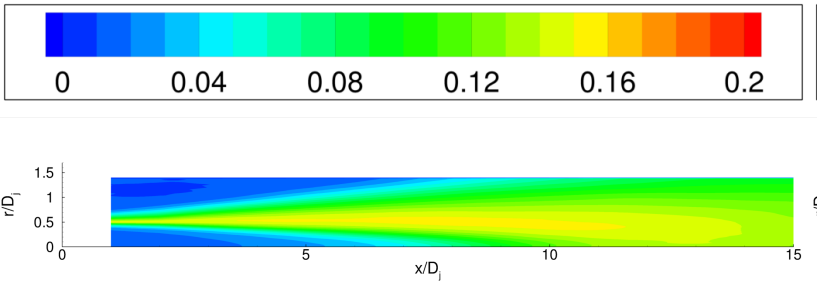

(a) Streamwise RMS Velocity (Exp)

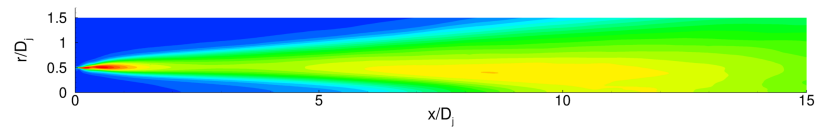

(c) Streamwise RMS Velocity (LES)
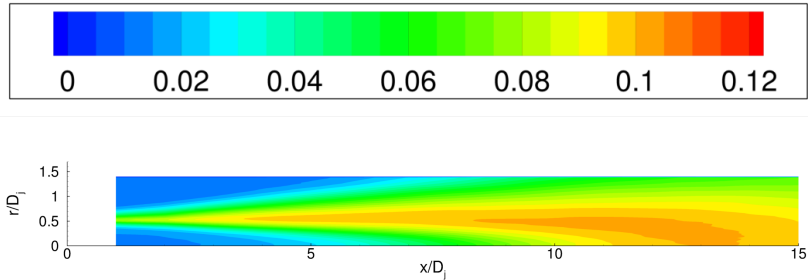

(b) Radial RMS Velocity (Exp)

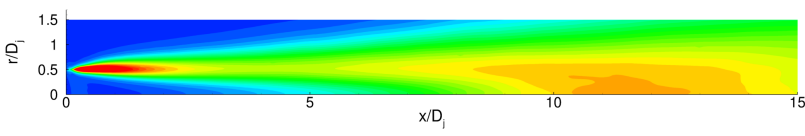

(d) Radial RMS Velocity (LES)

Figure 15: Experimental and LES axisymmetric jet turbulence from reference 115.

that reproduce correct Reynolds stresses: burst models, ${ }^{116}$ synthetic eddy methods, ${ }^{117}$ and digital filtering. ${ }^{118}$ Another method, used with wall boundary layers, involves a recycling technique whereby a section of the upstream boundary layer is simulated and the outflow of that region is rescaled and re-used at the inflow boundary to create a realistic inflow condition to the domain of interest. ${ }^{119,120}$ Care must be taken to ensure that the upstream region is not so short as to artificially limit the size of turbulent structures it allows. The recycling method can also be difficult to implement when the flow is not predominantly one-dimensional or when complex geometries are involved.

Reflecting waves from far-field and outflow boundaries can propagate back into the region of interest and contaminate a solution. Numerical boundary treatments constructed using characteristic theory can be used to reduce unwanted wave reflections. Alternatively, many simulations employ a "sponge layer" of grid cells to absorb the outgoing waves. By increasing the grid spacing in these outflow regions, the dissipation inherent in the numerical scheme will help to damp the outgoing waves. Sometimes additional explicit dissipation must be added along these boundaries.

\section{III.D. Sub-grid Modeling}

In LES, sub-grid scale models are used to account for the unresolved unsteady motion, which has a net dissipative effect on the resolved flow. Explicit sub-grid models are formulated to dissipate the appropriate amount of energy from the large-scale turbulence based upon the resolved motion in the simulation and the filter width, which is typically related to the grid size. Meneveau ${ }^{121}$ categorizes sub-grid models into three general categories: 1) eddy viscosity models, 2) Germano identity and dynamic models, and 3) similarity, nonlinear and deconvolution models. Eddy viscosity models, such as the original Smagorinsky ${ }^{122}$ model or Vremans model, ${ }^{123}$ are the most widely used models for free shear flows. Bogey and Bailly ${ }^{124}$ showed that the additional viscosity imposed through the sub-grid model lowered the effective Reynolds number of a jet flow, altering the turbulent structures and resultant jet noise. Sub-grid models based on categories 2 and 3 , such as the dynamic Smagorinsky model ${ }^{125}$ and the approximate deconvolution model ${ }^{126}$ are typically less dissipative and have shown good success.

A popular alternative to sub-grid modeling is implicit LES (ILES). ILES encompasses a wide variety of approaches that do not employ a traditional sub-grid model but instead rely upon the dissipation in the simulation's numerics to dissipate the large-scale turbulence. A popular subset of ILES methods is monotone integrated large-eddy simulation (MILES ${ }^{127}$ which employs monotone numerical methods such as Flux Corrected Transport (FCT), Monotone Upstream-centered Schemes for Conservation Laws (MUSCL), and Piecewise Parabolic Methods (PPM). In these methods the energy dissipation is implicit in the numerical scheme. Other ILES methods use centered difference methods and must rely on an explicitly added artificial dissipation term, such as a low pass filter. ${ }^{115,128}$ Results with any implicit LES method are obviously very dependent on the numerical scheme, but there are many excellent examples in the literature. Comparisons of implicit and explicit modeling using the same numerical method and grid show that the implicit method is much less dissipative. ${ }^{128}$ 


\section{III.E. Filtering}

Filtering separates the flow into large-scale resolved structures, that are directly resolved by the code, and small-scale unresolved structures which are modeled. Applying a filter to the Navier-Stokes equations is fundamental to the derivation of the LES equations. However, the need to explicitly filter the solution as part of the simulation process is less clear. Many researchers use the implicit filtering approach, arguing that the discretization process acts as a filter and no further filtering is necessary. ${ }^{83}$ Others explicitly apply a filter to the solution at each time step. Explicitly filtering the solution is the more rigorous approach and gives the user control over the filter function and width. But in many codes, the real benefit of filtering is to provide artificial dissipation for stability, since it removes the small unresolved (or under-resolved) waves that lead to instability. Successful simulations have been performed with both approaches.

\section{Future Research and Validation Needs}

The turbulence modeling community has periodically come together to evaluate turbulence models on a common set of problems in order to assess the current state of the art. A few well known gatherings include the 1968 AFOSR-IFP-Stanford Conference on the Computation of Turbulent Boundary Layers (often referred to as Stanford Olympics I), the 1972 Conference on Free Turbulent Shear Flows, and the 1980-81 AFOSR-HTTM-Stanford Conference on Complex Turbulent Flows (Stanford Olympics II). More recently, a number of AIAA workshops have been held on the topics of drag prediction, shock wave turbulent boundary interaction, and propulsion aerodynamics. These activities have helped to identify shortcomings of existing turbulence models as well as problems with the way certain turbulence models have been implemented in different codes, including in some cases additional model corrections that were used by default but not fully documented. There is now an AIAA Turbulence Model Benchmarking Working Group which has a website at http://turbmodels.larc.nasa.gov/ devoted to disclosing model formulations, implementation issues, and verification cases. Users and developers now have a centralized source for verifying that their code and turbulence model will produce standardized results. The authors own work with other developers and codes have identified implementation issues that are sometimes problem specific. Due to the complexity of modern CFD codes, this type of collaboration is critically important and should continue.

One on-going problem with model development is locating quality data for use in calibration and validation. At present, there is no centralized repository for experimental data. While a few publishers now support the archival of on-line data, the amount of information one can include in a journal article is quite limited. NASA and/or AIAA should consider how to bring experimentalists together to analyze data for a series of fundamental cases relevant to turbulence model development, evaluate data quality and completeness, and if possible arrive at a set of consensus data that the modeling community can easily access.

Additional experimental data is needed to further improve understanding of turbulent mixing layers and jets and aid in model development. For incompressible mixing layers, additional investigations are needed to better understand the relationship between different mixing layer thickness definitions, the effect of density ratio on growth rate, and the variation of peak turbulence statistics in the self-similar region. For compressible mixing layers, additional data from multiple facilities are needed to assess the changes in turbulence anisotropy. Care should also be taken to gather the type of detailed data necessary for calibration and/or validation of future modeling techniques. This includes: controlled and well-documented inflow conditions including the turbulence state and statistics; examination of both the development and selfsimilar regions; and measurements beyond just the mean flow and shear stress. Future model development will require knowledge of the three components of velocity in order to evaluate the mean stress and strain rate tensors and each of the Reynolds stresses, as well as higher-order correlations that contribute to the Reynolds stress transport equation.

LES and DNS offer the most promise for providing "complete" flowfield information for use in RANS model development. As computational resources continue to advance and these methods are applied more frequently and at higher Reynolds numbers, the simulations should also be used as a means for evaluating RANS model approximations. Single-point correlations could be computed for the higher-order moments that are not readily available experimentally. These correlations could then be used to examine the validity of various model approximations throughout the flowfield (i.e., the Boussinesq approximation that relates the stress and strain rate tensors, the weak equilibrium assumption used in the formulation of algebraic Reynolds stress models, modeled forms of the pressure-strain correlation tensor, various gradient diffusion approximations, etc.). 


\section{Conclusions}

Turbulent free shear flows, particularly mixing layers and jets, play a vital role in aeropropulsion applications. Experimental observations, especially at low Reynolds number, indicate the presence of large-scale coherent structures whose formation is triggered by the growth of instabilities in the initial part of the shear layer. As the flow progresses downstream, secondary instabilities are triggered that lead to the formation of streamwise vortices. The interaction between these various turbulent structures cause a distortion of the flow, increased three-dimensionality, and vortex stretching that lead to the development of small scale turbulence. Far downstream, the flow reaches a fully developed state where the profiles of mean velocity and turbulence statistics achieve self-similarity when plotted using appropriately scaled variables. In this region, the shear layer grows linearly with downstream distance.

Reynolds-averaged Navier-Stokes (RANS) methods are widely used in production CFD, and are frequently able to capture trends exhibited by experiments when the geometry or flow conditions are altered. However, the accuracy of existing turbulence models still leaves much to be desired. These models fail to capture a number of basic, yet critical, flow features including: differences between planar and axisymmetric shear flows, sensitivity to compressibility effects on the flow, and temperature effects on shear layer growth. Attempts to resolve some of these issues by adding explicit dilatation terms, vortex stretching terms, and temperature corrections have had only limited success. There is ample experimental evidence that many of these phenomena are accompanied by changes in the organization of the large-scale turbulent structures. As such, RANS modeling efforts will likely need to move beyond simple eddy viscosity models which primarily affect the flow through changes in the turbulent shear stress. Reynolds stress models might provide a better representation of these flows since they bypass the linear stress-strain relationship imposed by the Boussinesq approximation used in eddy viscosity models, allow for turbulence anisotropy, and account for changes in the pressure-strain correlation tensor. It might be possible to further extend these models by making the closure coefficients sensitive to local flow invariants.

Large-eddy simulation (LES) methods do not rely upon the numerous modeling approximations used by RANS models. Instead, the unsteady large-scale turbulent motion is resolved on the computational mesh and only the smaller scale turbulence is modeled. These methods naturally retain more of the physics described by the Navier-Stokes equations. However, LES is still subject to some uncertainty in physical modeling. Correctly specifying the unsteady inflow conditions is crucial for free shear flow simulations. How those conditions should best be approximated from only mean or statistical data remains an unknown. The method used to model the sub-grid scale contributions is also very important, whether that is done implicitly by the inherent dissipation in the numerical scheme or through explicit model terms and coefficients. LES is also sensitive to a number of numerical issues including: the dissipation of the numerical scheme, the use of explicit filtering particularly on non-uniform grids, and the resolution and quality of the computational mesh. The high-order numerical schemes often used for LES do not lend themselves well to complex grid topologies or unstructured grids. Current limitations in computational resources restrict the practical viability of LES methods to low Reynolds number flows. For free shear flow applications, adequate resolution of the smallscale turbulence in the initial part of the shear layer remains a challenge. As advances in computational resources allow for higher resolution grids and higher Reynolds number simulations, LES should become a more widely used tool for CFD simulations.

\section{References}

${ }^{1}$ Goebel, S. and Dutton, J., "Experimental Study of Compressible Turbulent Mixing Layers," AIAA Journal, Vol. 29, No. 4, April 1991, pp. 538-546.

${ }^{2}$ Görtler, H., "Berechnung von Aufgaben der freien Turbulenz auf Grund eines neuen Näherungsansatzes," Zeitschrift fur Angewandte Mathematik und Mechanik, Vol. 22, No. 5, Oct. 1942, pp. 244-254.

${ }^{3}$ Papamoschou, D. and Roshko, A., "The Compressible Turbulent Shear Layer: An Experimental Study," Journal of Fluid Mechanics, Vol. 197, 1988, pp. 453-477.

${ }^{4}$ Samimy, M. and Elliot, G., "Effects of Compressibility on the Characteristics of Free Shear Layers," AIAA Journal, Vol. 28, No. 3, March 1990, pp. 439-445.

${ }^{5}$ Brown, G. and Roshko, A., "On Density Effects and Large Structure in Turbulent Mixing Layers," Journal of Fluids Mechanics, Vol. 64, 1974, pp. 775-816. 2005.

${ }^{6}$ Yoder, D. A., Algebraic Reynolds Stress Modeling of Planar Mixing Layer Flows, Ph.D. thesis, University of Cincinnati,

${ }^{7}$ Liepmann, H. W. and Laufer, J., "Investigations of Free Turbulent Mixing," NACA TN-1257, Aug. 1947. 
${ }^{8}$ Sabin, C. M., "An Analytical and Experimental Study of the Plane, Incompressible, Turbulent Free-Shear Layer with Arbitrary Velocity Ratio and Pressure Gradient," AFOSR TN-5443, U.S. Air Force, Oct. 1963.

${ }^{9}$ Yakovlevskiy, O. V., "The Problem of the Thickness of the Turbulenct Mixing on the Boundary Between Two Gas Streams of Different Velocity and Density," Izv. Akad. Nauk SSSR, Otd. Tekhn. Nauk, Vol. 10, 1958, See Abramovich (1963) and Birch and Eggers (1972).

${ }^{10}$ Zhestkov, B. A., Glazkov, V. V., and Guseva, M. D., See Abramovich (1963) and Birch and Eggers (1972).

${ }^{11}$ Miles, J. B. and Shih, J.-s., "Similarity Parameter for Two-Stream Turbulent Jet-Mixing Region," AIAA Journal, Vol. 6 , No. 7, July 1968, pp. 1429-1430.

${ }^{12}$ Mills, R. D., "Numerical and Experimental Investigations of the Shear Layer Between Two Parallel Streams," Journal of Fluid Mechanics, Vol. 33, No. 3, Sept. 1968, pp. 591-616, See also Birch and Eggers (1972).

${ }^{13}$ Wygnanski, I. and Fiedler, H. E., "The Two-Dimensional Mixing Region," Journal of Fluid Mechanics, Vol. 41, No. 2, April 1970, pp. 327-361.

${ }^{14}$ Spencer, B. W. and Jones, B. G., "Statistical Investigation of Pressure and Velocity Fields in the Turbulent Two-Stream Mixing Layer," AIAA Paper 71-0613, June 1971.

${ }^{15}$ Yule, A. J., "The Spreading of Turbulent Mixing Layers," AIAA Journal, Vol. 10, No. 5, May 1972, pp. 686-687.

${ }^{16}$ Patel, R. P., "An Experimental Study of a Plane Mixing Layer," AIAA Journal, Vol. 11, No. 1, Jan. 1973 , pp. 67-71.

${ }^{17}$ Champagne, F. H., Pao, Y. H., and Wygnanski, I. J., "On the Two-Dimensional Mixing Region," Journal of Fluid Mechanics, Vol. 74, No. 2, 1976, pp. 209-250.

${ }^{18}$ Batt, R. G., "Turbulent Mixing of Passive and Chemically Reacting Species in a Low-Speed Shear Layer," Journal of Fluid Mechanics, Vol. 82, No. 1, 1977, pp. 53-95.

${ }^{19}$ Browand, F. K. and Latigo, B. O., "Growth of the Two-Dimensional Mixing Layer from a Turbulent and Nonturbulent Boundary Layer," Physics of Fluids, Vol. 22, No. 6, June 1979, pp. 1011-1019.

${ }^{20}$ Pui, N. K. and Gartshore, I. S., "Measurements of the Growth Rate and Structure in Plane Turbulent Mixing Layers," Journal of Fluid Mechanics, Vol. 91, March 1979, pp. 111-130.

${ }^{21}$ Oster, D. and Wygnanski, I., "The Forced Mixing Layer Between Parallel Streams," Journal of Fluid Mechanics, Vol. 123, 1982, pp. 91-130.

${ }^{22}$ Weisbrot, I., Einav, S., and Wygnanski, I., "The Non-Unique Rate of Spread of the Two-Dimensional Mixing Layer," Physics of Fluids, Vol. 25, Oct. 1982, pp. 1691-1693.

${ }^{23}$ Dziomba, B. and Fiedler, H. E., "Effect of Initial Conditions on Two-Dimensional Free Shear Layers," Journal of Fluid Mechanics, Vol. 152, 1985, pp. 419-442.

${ }^{24}$ Bell, J. H. and Mehta, R. D., "Development of a Two-Stream Mixing Layer from Tripped and Untripped Boundary Layers," AIAA Journal, Vol. 28, Dec. 1990, pp. 2034-2042.

${ }^{25}$ Mehta, R. D., "Effect of Velocity Ratio on Plane Mixing Layer Development: Influence of the Splitter Plate Wake," Experiments in Fluids, Vol. 10, No. 4, 1991, pp. 194-204, Presented at the Seventh Symposium on Turbulent Shear Flows.

${ }^{26}$ Delville, Joël, La décomposition orthogonale aux valeurs propres et l'analyse de l'organisation tridimensionnelle des écoulements turbulents cisaillés libres, Ph.D. thesis, Université de Poitiers, 1995, See also AGARD Advisory Report No. 345 (1998).

${ }^{27}$ Olsen, M. G. and Dutton, J. C., "Stochastic Estimation of Large Structures in an Incompressible Mixing Layer," $A I A A$ Journal, Vol. 40, No. 12, Dec. 2002, pp. 2431-2438.

${ }^{28}$ Baker, R. L. and Weinstein, H., "Experimental Investigation of the Mixing of Two Parallel Streams of Dissimilar Fluids," NASA CR-957, Jan. 1968.

${ }^{29}$ Abramovich, G. N., Yakovlevsky, O. V., Smirnova, I. P., Secundov, A. N., and Krasheninnikov, S. Y., "An Investigation of the Turbulent Jets of Different Gases in a General Stream," Astronautica Acta, Vol. 14, No. 3, March 1969, pp. 229-240.

${ }^{30}$ Brown, J. L., Heterogeneous Turbulent Mixing Layer Investigations Utilizing a 2-D 2-Color Laser Doppler Anemometer and a Concentration Probe, Ph.D. thesis, University of Missouri-Columbia, 1978.

${ }^{31}$ Bogdanoff, D. W., "Interferometric Measurement of Heterogeneous Shear-Layer Spreading Rates," AIAA Journal, Vol. 22, No. 11, Nov. 1984, pp. 1550-1555.

${ }^{32}$ Goebel, S. G., An Experimental Study of Compressible Turbulent Mixing Layers, Ph.D. thesis, University of Illinois at Urbana-Champaign, 1990.

${ }^{33}$ Rogers, M. M. and Moser, R. D., "Direct Simulation of a Self-Similar Turbulent Mixing Layer," Physics of Fluids, Vol. 6, No. 2, 1994, pp. 903-923.

${ }^{34}$ Pantano, C. and Sarkar, S., "A Study of Compressibility Effects in the High-Speed Turbulent Shear Layer Using Direct Simulation," Journal of Fluid Mechanics, Vol. 451, 2002, pp. 329-371.

${ }^{35}$ Batt, R. G., "Some Measurements on the Effect of Tripping the Two-Dimensional Shear Layer," AIAA Journal, Vol. 13, No. 2, Feb. 1975, pp. 245-247.

${ }^{36}$ Winant, C. D. and Browand, F. K., "Vortex Pairing: The Mechanism of Turbulent Mixing Layer Growth at Moderate Reynolds Number," Journal of Fluid Mechanics, Vol. 63, 1974, pp. 237-255.

${ }^{37}$ Bernal, L. P. and Roshko, A., "Streamwise Vortex Structure in Plane Mixing Layers," Journal of Fluid Mechanics, Vol. 170, 1986, pp. 499-525.

${ }^{38}$ Dutton, J. C., "Compressible Turbulent Free Shear Layers," Turbulence in Compressible Flows, AGARD-R-819, 1997, pp. 2.1-2.42.

${ }^{39}$ Jimenez, J., Cogollos, M., and Bernal, L. P., "A Perspective View of the Plane Mixing Layer," Journal of Fluid Mechanics, Vol. 152, 1985, pp. 125-143.

${ }^{40}$ Breidenthal, R. E., "Structure in Turbulent Mixing Layers and Wakes Using a Chemical Reactions," Journal of Fluid Mechanics, Vol. 125, 1981, pp. 397-409. 
${ }^{41}$ Maydew, R. C. and Reed, J. F., "Turbulent Mixing of Compressible Free Jets," AIAA Journal, Vol. 1, No. 6, 1963, pp. $1443-1444$.

${ }^{42}$ Ikawa, H. and Kubota, T., "Investigation of Supersonic Turbulent Mixing Layer with Zero Pressure Gradient," AIAA Journal, Vol. 13, No. 5, May 1975, pp. 566-572, Presented as AIAA Paper 74-40.

${ }^{43}$ Chinzei, N., Masuya, G., Komuro, T., Murakami, A., and Kudou, K., "Spreading of Two-Stream Supersonic Turbulent Mixing Layers," Physics of Fluids, Vol. 29, No. 5, May 1986, pp. 1345-1347.

${ }^{44}$ Messersmith, N. L., Goebel, S. G., Renie, J. P., Dutton, J. C., and Krier, H., "Investigation of Supersonic Mixing Layers," Journal of Propulsion and Power, Vol. 6, No. 4, July 1990, pp. 353-354, Presented as AIAA Paper 88-0702.

${ }^{45}$ Elliott, G. and Samimy, M., "Compressibility Effects in Free Shear Layers," Physics of Fluids A, Vol. 2, No. 7, 1990, pp. 1231-1240.

${ }^{46}$ Fourgette, D. C., Mungal, M. G., Barlow, R. S., and Dibble, R. W., "Concentration Measurements in a Supersonic Shear Layer," AIAA Paper 91-0627, Jan. 1991.

${ }^{47}$ Fourgette, D. C., Mungal, M. G., and Dibble, R. W., "Time Evolution of the Shear Layer of a Supersonic Axisymmetric Jet," AIAA Journal, Vol. 29, No. 7, 1991, pp. 1123-1129, See also AIAA Paper 90-0508.

${ }^{48}$ Gruber, M. R., Messersmith, N. L., and Dutton, J. C., "Three-Dimensional Velocity Field in a Compressible Mixing Layer," AIAA Journal, Vol. 31, No. 11, Nov. 1993, pp. 2061-2067.

${ }^{49}$ Hall, J. L., Dimotakis, P. E., and Rosemann, H., "Experiments in Nonreacting Compressible Shear Layers," AIAA Journal, Vol. 31, No. 12, Dec. 1993, pp. 2247-2253.

${ }^{50}$ Debisschop, J. R., Chambres, O., and Bonnet, J. P., "Velocity Field Characteristics in Supersonic Mixing Layers," Experimental Thermal and Fluid Science, Vol. 9, 1994, pp. 147-155.

${ }^{51}$ Clemens, N. and Mungal, M., "Large-Scale Structure and Entrainment in the Supersonic Mixing Layer," Journal of Fluid Mechanics, Vol. 284, 1995, pp. 171-216.

${ }^{52}$ Chambres, O., Analyse Expérimentale de la Modélisation de la Turbulence en Couche de mélange Supersonique, Ph.D. thesis, Université de Poitiers, 1997.

${ }^{53}$ Rossmann, T., An Experimental Investigation of High Compressibility Mixing Layers, Ph.D. thesis, Stanford University, 2001.

${ }^{54}$ Olsen, M. G. and Dutton, J. C., "Planar Velocity Measurements in a Weakly Compressible Mixing Layer," Journal of Fluid Mechanics, Vol. 486, 2003, pp. 51-77.

${ }^{55}$ Vreman, A., Sandham, N., and Luo, K., "Compressible Mixing Layer Growth Rate and Turbulence Characteristics," Journal of Fluid Mechanics, Vol. 320, 1996, pp. 235-258.

${ }^{56}$ Day, M. J. and Reynolds, W. C., "The Structure of the Compressible Reacting Mixing Layer: Insights from Linear Stability Analysis," Physics of Fluids, Vol. 10, No. 4, 1998, pp. 993-1007.

${ }^{57}$ Birch, S. F. and Eggers, J. M., "A Critical Review of the Experimental Data for Developed Free Turbulent Shear Layers," Free Turbulent Shear Flows, Vol. I, NASA SP-321, 1972, pp. 11-40.

${ }^{58}$ Urban, W. D. and Mungal, M. G., "Planar Velocity Measurements in Compressible Mixing Layers," Journal of Fluid Mechanics, Vol. 431, 2001, pp. 189-222.

${ }^{59}$ Clemens, N. and Mungal, M., "Two- and Three-Dimensional Effects in the Supersonic Mixing Layer," AIAA Journal, Vol. 30, No. 4, April 1992, pp. 973-981.

${ }^{60}$ Papamoschou, D., "Communication Paths in the Compressible Shear Layer," AIAA Paper 1990-0155, Jan. 1990.

${ }^{61}$ Papamoschou, D., "Zones of Influence in the Compressible Shear Layer," Fluid Dynamics Research, Vol. 11, 1993, pp. 217-228.

${ }^{62}$ Gutmark, E. J., Schadow, K. C., and Yu, K. H., "Mixing Enhancement in Supersonic Free Shear Flows," Annual Review of Fluid Mechanics, Vol. 27, 1995, pp. 375-417.

${ }^{63}$ Michalke, A., "Instability of a Compressible Circular Free Jet with Consideration of the Influence of the Jet Boundary Layer Thickness," Zeitschrift fuer Flugwissenschaften, Vol. 19, No. 9, Sept. 1971, pp. 319-328, See Also NASA TM-75190.

${ }^{64}$ Koshigoe, S., Tubis, A., and Ho, C.-M., "Vortex Deformation in Elliptic-Core Jets from the Perspective of Linear Instability Analysis," Physics of Fluids, Vol. 31, No. 9, Sept. 1988, pp. 2504-2517.

${ }^{65}$ Gutmark, E. J. and Grinstein, F. F., "Flow Control with Noncircular Jets," Annual Review of Fluid Mechanics, Vol. 31, 1999, pp. 239-272.

${ }^{66}$ Hussain, F. and Husain, H. S., "Elliptic Jets. Part 1. Characteristics of Unexcited and Excited Jets," Journal of Fluid Mechanics, Vol. 208, Nov. 1989, pp. 257-320.

${ }^{67}$ Zaman, K. B. M., "Axis Switching and Spreading of an Asymmetric Jet: The Role of Coherent Structure Dynamics," Journal of Fluid Mechanics, Vol. 316, June 1996, pp. 1-27.

${ }^{68}$ Lau, J. C., "Mach Number and Temperature Effect on Jets," AIAA Journal, Vol. 18, No. 6, June 1980, pp. 609-610.

${ }^{69}$ Lepicovsky, J., "Total Temperature Effects on Centerline Mach Number Characteristics of Free Jets," AIAA Journal, Vol. 28, No. 3, March 1990, pp. 478-482.

${ }^{70}$ Monkewitz, K., "Absolute Instability in Hot Jets," AIAA Journal, Vol. 26, No. 8, Aug. 1988, pp. 911-916.

${ }^{71}$ Monkewitz, P. A., Bechert, D. W., Barsikow, B., and Lehmann, B., "Self-Excited Oscillations and Mixing in a Heated Round Jet," Journal of Fluid Mechanics, Vol. 213, April 1990, pp. 611-639.

${ }^{72}$ Witze, P. O., "Centerline Velocity Decay of Compressible Free Jets," AIA A Journal, Vol. 12, No. 4, April 1974, pp. 417418.

${ }^{73}$ Bridges, J. E. and Wernet, M. P., "The NASA Subsonic Jet Particle Image Velocimetry (PIV) Dataset," NASA TM2011-216807, Nov. 2011.

${ }^{74}$ Townsend, A. A., The Structure of Turbulent Shear Flow, Cambridge University Press, Cambridge, England, 2nd ed., 1976. 
${ }^{75}$ Bradshaw, P., "Turbulence Modeling with Application to Turbomachinery," Progress in Aerospace Sciences, Vol. 32, 1996, pp. 575-624. 1992.

${ }^{76}$ Spalart, P. and Allmaras, S., "A One-Equation Turbulence Model for Aerodynamic Flows," AIAA Paper 92-0439, Jan.

${ }^{77}$ Launder, B. and Sharma, B., "Application of the Energy-Dissipation Model of Turbulence to the Calculation of Flow Near a Spinning Disc," Letters in Heat and Mass Transfer, Vol. 1, 1974, pp. 131-138.

${ }^{78}$ Bradbury, L. J. S., "The Structure of a Self-Preserving Turbulent Plane Jet," Journal of Fluid Mechanics, Vol. 23, No. 1, Sept. 1965, pp. 31-64.

${ }^{79}$ Wygnanski, I. and Fiedler, H. E., "Some Measurements in the Self-Preserving Jet," Journal of Fluid Mechanics, Vol. 38, No. 3, Sept. 1969, pp. 577-612.

${ }^{80}$ Witze, P. O. and Dwyer, H. A., "The Turbulent Radial Jet," Journal of Fluid Mechanics, Vol. 75, No. 3, June 1976, pp. 401-417.

${ }^{81}$ Fage, A. and Falkner, V. M., "Note on Experiments on the Temperature and Velocity in the Wake of a Heated Cylindrical Obstacle," Proceedings of the Royal Society of London Series A, Mathematical and Physical Sciences, Vol. 135, No. 828, April 1932, pp. 702-705.

${ }^{82}$ Wilcox, D. C., Turbulence Modeling for CFD, DCW Industries, 3rd ed., 2006.

${ }^{83}$ Wilcox, D. C., Turbulence Modeling for CFD, DCW Industries, 2nd ed., 1998.

${ }^{84}$ Wilcox, D., "Reassessment of the Scale-Determining Equation for Advanced Turbulence Models," AIAA Journal, Vol. 26, No. 11, Nov. 1988, pp. 1299-1310.

${ }^{85}$ Menter, F. R., "Two-Equation Eddy-Viscosity Turbulence Models for Engineering Applications," AIAA Journal, Vol. 32, No. 8, Aug. 1994, pp. 1598-1605, Presented as AIAA Paper 93-2906.

${ }^{86}$ Pope, S., "An Explaination of the Turbulent Round-Jet/Plane-Jet Anomaly," AIAA Journal, Vol. 16, No. 3, Feb. 1978, pp. 279-281.

${ }^{87}$ Robinson, D., Harris, J., and Hassan, H., "A Unified Turbulence Closure Model for Asisymmetric and Planar Free Shear Flows," AIA A Journal, Vol. 33, No. 12, Dec. 1995, pp. 2325-2331, Presented as AIAA Paper 95-0360.

${ }^{88}$ Speziale, C. G., Abid, R., and Anderson, E. C., "A Critical Evaluation of Two-Equation Models for Near Wall Turbulence," NASA CR-182068, June 1990, See also ICASE Report No. 90-46.

${ }^{89}$ Bardina, J., Huang, P., and Coakley, T., "Turbulence Modeling Validation, Testing, and Development," NASA TM110446, April 1997.

${ }^{90}$ Birch, Stanley, F., "Comment on "Computation of Turbulent Axisymmetric and Nonaxisymmetric Jet Flows Using the $k-\epsilon$ Model"," AIAA Journal, Vol. 35, No. 4, April 1997, pp. 760-761.

${ }^{91}$ Birch, Stanley, F., "On the Role of Structure in Turbulent Mixing," AIAA Paper 97-2636, Jan. 1997.

${ }^{92}$ Pope, S., "A More Effective Viscosity Hypothesis," Journal of Fluid Mechanics, Vol. 72, 1975, pp. 331-340.

${ }^{93}$ Sarkar, S., Erlebacher, G., Hussaini, M. Y., and Kreiss, H. O., "The Analysis and Modeling of Dilatational Terms in Compressible Turbulence," Journal of Fluid Mechanics, Vol. 227, 1991, pp. 473-493.

${ }^{94}$ Sarkar, S., "The Pressure-Dilatation Correlation in Compressible Flows," Physics of Fluids A, Vol. 4, No. 12, 1992, pp. 2674-2682.

${ }^{95}$ Dembowski, M. A. and Georgiadis, N. J., "An Evaluation of Parameters Influencing Jet Mixing Using the WIND Navier-Stokes Code," NASA TM-211727, Aug. 2002.

${ }^{96}$ Seiner, J. M., Ponton, M. K., Jansen, B. J., and Lagan, N. T., "The Effects of Temperature on Supersonic Jet Noise Emission," AIAA Paper 92-02-046, May 1992.

${ }^{97}$ Sarkar, S., "The Stabilizing Effect of Compressibility in Turbulent Shear Flow," Journal of Fluid Mechanics, Vol. 282, 1995, pp. 163-186.

${ }^{98}$ Freund, J. B., Lele, S. K., and Moin, P., "Compressibility Effects in a Turbulent Annular Mixing Layer. Part 1. Turbulence and Growth Rate," Journal of Fluid Mechanics, Vol. 421, 2000, pp. 229-267.

${ }^{99}$ Hamba, F., "Effects of Pressure Fluctuations on Turbulence Growth in Compressible Homogeneous Shear Flow," Physics of Fluids, Vol. 11, No. 6, June 1997, pp. 1623-1635.

${ }^{100}$ Fujiwara, H., Matsuo, Y., and Arakawa, C., "A Turbulence model for the Pressure-Strain Correlation Term Accounting for Compressibility Effects," International Journal of Heat and Fluid Flow, Vol. 21, 2000, pp. 354-358.

${ }^{101}$ Huang, S. and Fu, S., "Modelling of Pressure-Strain Correlation in Compressible Turbulent Flow," Acta Mech Sin, Vol. 24, 2008, pp. 37-43.

${ }^{102}$ Gomez, C. A. and Girimaji, S. S., "Algebraic Reynolds Stress Model (ARSM) for Compressible Shear Flows," AIAA Paper 2011-3572, June 2011.

${ }^{103}$ Georgiadis, N. J. and DeBonis, J. R., "Navier-Stokes Analysis Methods for Turbulent Jet Flows with Application to Aircraft Exhaust Nozzles," Progress in Aerospace Sciences, Vol. 42, 2006, pp. 377-418.

${ }^{104}$ Georgiadis, N. J., Yoder, D. A., Vyas, M. A., and Engblom, W. A., "Status of Turbulence Modeling for Hypersonic Propulsion Flowpaths," NASA TM-2012-217277, April 2012.

${ }^{105}$ Burrows, M. and Kurkov, A., "Analytical and Experimental Study of Supersonic Combustion of Hydrogen in a Vitiated Airstream," NASA TM-X-2028, Sept. 1973.

${ }^{106}$ Nagano, Y. and Kim, C., "A Two-Equation Model for Heat Transport in Wall Turbulent Shear Flows," Journal of Heat Transfer, Vol. 110, Aug. 1988, pp. 583-589.

${ }^{107}$ Sommer, T., So, R., and Zhang, H., "Supersonic Flow Calculations Using a Reynolds-Stress and a Thermal Eddy Diffusivity Turbulence Model," Journal of Fluids Engineering, Vol. 116, Sept. 1994, pp. 469-476.

${ }^{108}$ Brinckman, K. W., Calhoon, W. H. J., and Dash, S. M., "Scalar Fluctuation Modeling for High-Speed Aeropropulsive Flows," AIAA Journal, Vol. 45, No. 5, May 2007, pp. 1036-1046. 
${ }^{109}$ Xiao, X., Hassan, H. A., and Baurle, R. A., "Modeling Scramjet Flows with Variable Turbulent Prandtl and Schmidt Numbers," AIAA Journal, Vol. 45, No. 6, June 2007, pp. 1415-1423, See also AIAA Paper 2006-0128.

${ }^{110}$ Tam, C. K. W. and Webb, J. C., "Dispersion Relation-Preserving Finite Difference Schemes for Computational Aeroacoustics," Journal of Computational Physics, Vol. 107, No. 2, Aug. 1993, pp. 262-281.

${ }^{111}$ Bogey, C. and Bailly, C., "A Family of Low Dispersive and Low Dissipative Explicit Schemes for Flow and Noise Computations," Journal of Computational Physics, Vol. 194, No. 1, Feb. 2004, pp. 194-214.

${ }^{112}$ Lele, S. K., "Compact Finite Difference Schemes with Spectral-Like Resolution," Journal of Computational Physics, Vol. 103, No. 1, Nov. 1992, pp. 16-42.

${ }^{113}$ Ham, F., Mattsson, K., and Iaccarino, G., "Accurate and Stable Finite Volume Operators for Unstructured Flow Solvers," Center for Turbulence Research Annual Research Briefs, Stanford University, 2006, pp. 243-261.

${ }^{114}$ Cook, A. and Cabot, W., "Hyperviscosity for Shock-Turbulence Interactions," Journal of Computational Physics, Vol. 203, No. 2, March 2005, pp. 379-385.

${ }^{115}$ DeBonis, J., "A High-Resolution Capability for Large-Eddy Simulation of Jet Flows," AIAA Paper 2010-5023, June 2010.

${ }^{116}$ Batten, P., Goldberg, U., Kang, E., and Chakravarthy, S., "Smart Sub-Grid-Scale Models for LES and Hybrid RANS/LES," AIAA Paper 2011-3472, June 2011.

${ }^{117}$ Jarrin, N., Prosser, R., Uribe, J.-C., Benhamadouche, S., and Laurence, D., "Reconstruction of Turbulent Fluctuations for Hybrid RANS/LES Simulations using a Synthetic-Eddy Method," International Journal of Heat and Fluid Flow, Vol. 6, 2009 , pp. 435-442.

${ }^{118}$ di Mare, L., Klien, M., Jones, W. P., and Janicka, J., "Synthetic Turbulence Inflow Conditions for Large-Eddy Simulation," Physics of Fluids, Vol. 18, No. 2, 2006, pp. 1-11.

${ }^{119}$ Lund, T., Wu, X., and Squires, K., "Generation of Turbulent Inflow Data for Spatially-Developing Boundary Layer Simulations," Journal of Computational Physics, Vol. 140, No. 2, March 1998, pp. 233-258.

${ }^{120}$ Urbin, G. and Knight, D., "Large-Eddy Simulation of a Supersonic Boundary Layer Using an Unstructured Grid," AIAA Journal, Vol. 39, No. 7, 2001, pp. 1288-1295.

${ }^{121}$ Meneveau, C., "Turbulence: Subgrid-Scale Modeling," Scholarpedia, Vol. 5, No. 1, 2010, pp. 9489.

${ }^{122}$ Smagorinsky, J., "General Circulation Experiments with the Primitive Equations," Monthly Weather Review, Vol. 91, 1963, pp. 99-165.

${ }^{123}$ Vreman, A., "An Eddy-Viscosity Subgrid-Scale Model for Turbulent Shear Flow: Algebraic Theory and Applications," Physics of Fluids, Vol. 16, 2004, pp. 3570.

${ }^{124}$ Bogey, C. and Bailly, C., "Decrease of the Effective Reynolds Number with Eddy-Viscosity Subgrid-Scale Modeling," AIAA Journal, Vol. 43, No. 2, 2005, pp. 437-439.

${ }^{125}$ Moin, P., Squires, K., Cabot, W., and Lee, S., "A Dynamic Subgrid-Scale Model for Compressible Turbulence and Scalar Transport," Physics of Fluids A, Vol. 3, No. 11, 1991, pp. 2746-2757.

${ }^{126}$ Stolz, P. and Adams, N. A., "An Approximate Deconvolution Procedure for Large-Eddy Simulation," Physics of Fluids A, Vol. 11, No. 7, 1999, pp. 1699-1701.

${ }^{127}$ Grinstein, F. F., Margolin, L. G., and Rider, W. J., Implicit Large Eddy Simulation, Cambridge University Press, 2007.

${ }^{128}$ Bogey, C. and Bailly, C., "Computation of a High Reynolds Number Jet and its Radiated Noise Using Large Eddy Simulation Based on Explicit Filtering," Computers in Fluids, Vol. 35, No. 10, 2006, pp. 1344-1358. 



\begin{tabular}{|c|c|c|c|c|c|}
\hline \multicolumn{5}{|c|}{ REPORT DOCUMENTATION PAGE } & $\begin{array}{l}\text { Form Approved } \\
\text { OMB No. 0704-0188 }\end{array}$ \\
\hline \multicolumn{6}{|c|}{ 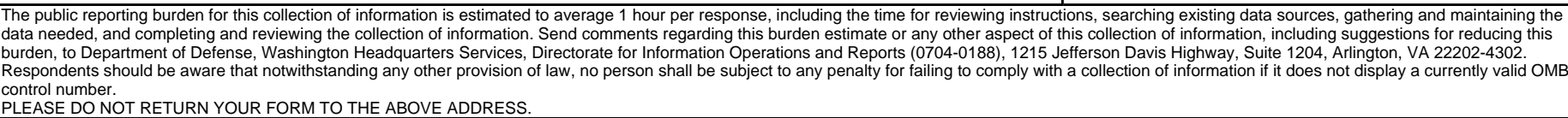 } \\
\hline \multicolumn{2}{|c|}{$\begin{array}{l}\text { 1. REPORT DATE (DD-MM-YYYY) } \\
01-08-2013\end{array}$} & \multicolumn{3}{|c|}{$\begin{array}{l}\text { 2. REPORT TYPE } \\
\text { Technical Memorandum }\end{array}$} & 3. DATES COVERED (From - To) \\
\hline \multirow{3}{*}{\multicolumn{5}{|c|}{$\begin{array}{l}\text { 4. TITLE AND SUBTITLE } \\
\text { Modeling of Turbulent Free Shear Flows }\end{array}$}} & 5a. CONTRACT NUMBER \\
\hline & & & & & 5b. GRANT NUMBER \\
\hline & & & & & 5c. PROGRAM ELEMENT NUMBER \\
\hline \multirow{3}{*}{\multicolumn{5}{|c|}{$\begin{array}{l}\text { 6. AUTHOR(S) } \\
\text { Yoder, Dennis, A.; DeBonis, James, R.; Georgiadis, Nicholas, J. }\end{array}$}} & 5d. PROJECT NUMBER \\
\hline & & & & & 5e. TASK NUMBER \\
\hline & & & & & $\begin{array}{l}\text { 5f. WORK UNIT NUMBER } \\
\text { WBS 794072.02.03.02.01 }\end{array}$ \\
\hline \multicolumn{5}{|c|}{$\begin{array}{l}\text { 7. PERFORMING ORGANIZATION NAME(S) AND ADDRESS(ES) } \\
\text { National Aeronautics and Space Administration } \\
\text { John H. Glenn Research Center at Lewis Field } \\
\text { Cleveland, Ohio 44135-3191 }\end{array}$} & $\begin{array}{l}\text { 8. PERFORMING ORGANIZATION } \\
\text { REPORT NUMBER } \\
\text { E-18747 }\end{array}$ \\
\hline \multirow{2}{*}{\multicolumn{5}{|c|}{$\begin{array}{l}\text { 9. SPONSORING/MONITORING AGENCY NAME(S) AND ADDRESS(ES) } \\
\text { National Aeronautics and Space Administration } \\
\text { Washington, DC 20546-0001 }\end{array}$}} & $\begin{array}{l}\text { 10. SPONSORING/MONITOR'S } \\
\text { ACRONYM(S) } \\
\text { NASA }\end{array}$ \\
\hline & & & & & $\begin{array}{l}\text { 11. SPONSORING/MONITORING } \\
\text { REPORT NUMBER } \\
\text { NASA/TM-2013-218072 }\end{array}$ \\
\hline \multicolumn{6}{|c|}{$\begin{array}{l}\text { 12. DISTRIBUTION/AVAILABILITY STATEMENT } \\
\text { Unclassified-Unlimited } \\
\text { Subject Categories: } 34 \text { and } 07 \\
\text { Available electronically at http://www.sti.nasa.gov } \\
\text { This publication is available from the NASA Center for AeroSpace Information, 443-757-5802 }\end{array}$} \\
\hline \multicolumn{6}{|c|}{ 13. SUPPLEMENTARY NOTES } \\
\hline \multicolumn{6}{|c|}{$\begin{array}{l}\text { 14. ABSTRACT } \\
\text { The modeling of turbulent free shear flows is crucial to the simulation of many aerospace applications, yet often receives less attention than } \\
\text { the modeling of wall boundary layers. Thus, while turbulence model development in general has proceeded very slowly in the past twenty } \\
\text { years, progress for free shear flows has been even more so. This paper highlights some of the fundamental issues in modeling free shear } \\
\text { flows for propulsion applications, presents a review of past modeling efforts, and identifies areas where further research is needed. Among } \\
\text { the topics discussed are differences between planar and axisymmetric flows, development versus self-similar regions, the effect of } \\
\text { compressibility and the evolution of compressibility corrections, the effect of temperature on jets, and the significance of turbulent Prandtl } \\
\text { and Schmidt numbers for reacting shear flows. Large eddy simulation greatly reduces the amount of empiricism in the physical modeling, } \\
\text { but is sensitive to a number of numerical issues. This paper includes an overview of the importance of numerical scheme, mesh resolution, } \\
\text { boundary treatment, sub-grid modeling, and filtering in conducting a successful simulation. }\end{array}$} \\
\hline \multicolumn{6}{|c|}{$\begin{array}{l}\text { 15. SUBJECT TERMS } \\
\text { Jet flow; Mixing layers; Turbolence models; Compressibility effects; Temperature effects; Propulsion }\end{array}$} \\
\hline \multicolumn{3}{|c|}{ 16. SECURITY CLASSIFICATION OF: } & $\begin{array}{l}\text { 17. LIMITATION OF } \\
\text { ABSTRACT }\end{array}$ & $\begin{array}{l}\text { 18. NUMBER } \\
\text { OF }\end{array}$ & $\begin{array}{l}\text { 19a. NAME OF RESPONSIBLE PERSON } \\
\text { STI Help Desk (email:help@sti.nasa.gov) }\end{array}$ \\
\hline $\begin{array}{l}\text { a. REPORT } \\
\text { U }\end{array}$ & $\begin{array}{l}\text { b. ABSTRACT } \\
\text { U }\end{array}$ & $\begin{array}{l}\text { c. THIS } \\
\text { PAGE } \\
\text { U }\end{array}$ & UU & $\begin{array}{l}\text { PAGES } \\
38\end{array}$ & $\begin{array}{l}\text { 19b. TELEPHONE NUMBER (include area code) } \\
443-757-5802\end{array}$ \\
\hline
\end{tabular}



